\title{
The Trace Formula for Schrödinger Operators on the Line
}

\author{
Walter Craig
}

Dept. of Mathematics, Stanford University, Stanford, CA 94305, USA *

Abstract. This paper discusses certain aspects of the spectral and inverse spectral problems for the Schrödinger operator $L(q)=-\frac{d^{2}}{d x^{2}}+q(x)$, for $q(x) \in C(\mathbb{R})$, the space of bounded continuous functions. The trace formula of the title is the relation

$$
q(0)=\lambda_{0}+\sum_{j=1}^{\infty}\left(\lambda_{2 j}+\lambda_{2 j-1}-2 \mu_{j}\right)
$$

with appropriate choices of $\left\{\lambda_{j}\right\}_{j=0}^{\infty}$ and $\left\{\mu_{j}\right\}_{j=1}^{\infty}$, which is a familiar relation in the theory of Hill's equation. We characterize the set $\mathscr{T} \subseteq C(\mathbb{R})$ of potentials for which this holds. Further extensions of the theory of Hill's equation are also obtained. From the spectrum $\sigma(L(q))$ a torus $T(q)$ is constructed, which is in general infinite dimensional; every $q(x) \in \mathscr{T}$ can be mapped to a continuous path on $T(q)$, described by the auxiliary spectrum $\left\{\mu_{j}\right\}_{j=1}^{\infty}$. Under certain geometrical conditions on $\sigma(L(q))$ this path is the orbit of a $C^{1}$ vector field on $T(q)$, and the mapping extends to one from the hull $\mathscr{H}(q)=\overline{\{q(x+\xi) ; \xi \in \mathbb{R}\}}$ to the closure of this orbit. In particular $\mathscr{H}(q)$ is compact. These results have applications in the theory of Schrödinger operators with ergodic potentials.

\section{Introduction}

This paper is concerned with the study of the spectral problem for the Schrödinger operator

$$
\left(-\frac{d^{2}}{d x^{2}}+q(x)\right) \psi=\lambda \psi,
$$

considered on the line $-\infty<x<+\infty$. We consider potentials $q(x) \in C(\mathbb{R})$, the space of bounded continuous functions. Two standard spectral problems are that

* Research partially supported by the Air Force Office of Scientific Research, the Office of Naval Research, and the National Science Foundation

Current address: Mathematics Department, Brown University Providence, RI 02912, USA 
of defining the operator on a dense subdomain of $L^{2}(\mathbb{R})$, and of defining the operator with additional Dirichlet conditions imposed at $x=0$. It is well known that these problems are of Weyl limit point case at $x \rightarrow \pm \infty$. Denote these two operators $L(q)$ and $L_{D}(q)$ respectively; their difference has a finite trace in the generalized sense, which we denote $\operatorname{tr}\left(L(q)-L_{D}(q)\right)$.

In the case that $q(x)$ is periodic, say of period 1 , this trace can be computed in two ways. Let $\left\{\lambda_{j}\right\}_{j=0}^{\infty}$ be the collection of periodic and anti-periodic eigenvalues of (1.1) on the interval $[0,1]$, and $\left\{\mu_{j}\right\}_{j=1}^{\infty}$ the collection of Dirichlet eigenvalues on the same interval (the auxiliary spectrum); it is a beautiful formula that

$$
\lambda_{0}+\sum_{j=1}^{\infty} \lambda_{2 j}+\lambda_{2 j-1}-2 \mu_{j}=2 \operatorname{tr}\left(L(q)-L_{D}(q)\right)=q(0) .
$$

The motivation of this paper is to extend this simple formula, with a suitable definition of $\lambda_{j}$ and $\mu_{j}$ to a larger class of potentials $q(x) \in C(\mathbb{R})$, extending certain results of Bargmann [2], McKean et al. [13, 14], Dubrovin et al. [6], Moser [15], Levitan [12], and Kotani and Krishna [10]. In particular, certain examples of Moser [16] and Avron and Simon [1] with nowhere dense spectra are included within this class. It turns out that the right-hand equality of (1.2) holds generally, for any $q(x) \in C(\mathbb{R})$; this is the result of Sect. 2. Furthermore, the trace of the difference of higher powers of $L(q), L_{D}(q)$ is computed, and is shown to be finite depending only on the local regularity of $q(x)$ near zero. The method uses the Feynman Kac expression for the heat kernel.

The left-hand equality of (1.2) will involve the spectrum. The simple formula cannot be true in general; however, it will hold for a certain class $\mathscr{T}$ of potentials $q(x)$ which we will identify by the behavior of the Green's function for $L(q)$ on the real axis. In particular, all periodic potentials belong in this class. We call this class "reflectionless" or "of Bargmann type" by analogy to the reflectionless multisoliton potentials of [2]. A discussion of this condition appears in Sect. 3, with the proof of the second equality of (1.2) in Theorem 3.6. The proper definition of the points $\lambda_{j}$ is as endpoints of spectral gaps for $L(q)$. The spectrum $\sigma(L(q))$ is a closed set of $\mathbb{R}$, with a finite minimum point $\lambda_{0}$, whose compliment consists of the ray $\left(-\infty, \lambda_{0}\right)$ union possible infinitely many open intervals (components of $\mathbb{R}-\sigma(L(q)))$ which we enumerate somehow, and denote $\left(\lambda_{2 j-1}, \lambda_{2 j}\right), j \in \mathbb{Z}^{+}$. This gives the $\lambda$ 's. Roughly, $\mu_{j} \in\left[\lambda_{2 j-1}, \lambda_{2 j}\right], j \in \mathbb{Z}^{+}$, are taken to be $L^{2}$ eigenvalues of the left and right half-line problems, combined in the operator $L_{D}(q)$. These points are of course not necessarily able to be enumerated in order on the line. The impact of the defining condition of $\mathscr{T}$ is that for this class the contributions of the continuous spectrum to the trace formula (1.2) cancel.

Via the trace formula one is naturally let to study the inverse spectral problem, considering $\left\{\lambda_{j}\right\}_{j=0}^{\infty}$ and $\left\{\mu_{j}\right\}_{j=1}^{\infty}$ as spectral data. Let $\mu_{j}(\xi)$ be as above, with respect to the half-line eigenvalue problem starting or ending at $\xi \in \mathbb{R}$. The trace formula recovers $q(\xi)$ from $\left\{\lambda_{j}\right\}$ and $\left\{\mu_{j}(\xi)\right\}$. As in the periodic case it is better to consider these spectral quantities in angular variables. Define a change of variables

$$
\cos \varphi_{j}=\frac{2 \mu_{j}}{\lambda_{2 j}-\lambda_{2 j-1}}-\frac{\lambda_{2 j}+\lambda_{2 j-1}}{\lambda_{2 j}-\lambda_{2 j-1}}
$$


with a choice of arccosine to be decided later; the set $\left\{\boldsymbol{\varphi} ; 0 \leqq \varphi_{j}<2 \pi, j \in \mathbb{Z}^{+}\right\}$gives global coordinates to an infinite dimensional torus $T$. This torus is compact in the topology specified by the distance function

$$
\|\varphi-\theta\|=\sup _{j \in \mathbb{Z}^{+}}\left(\lambda_{2 j}-\lambda_{2 j-1}\right)^{1 / 2}\left|\varphi_{j}-\theta\right|_{\bmod 2 \pi} .
$$

Define the function

$$
Q(\mu)=\lambda_{0}+\sum_{j=1}^{\infty} \lambda_{2 j}+\lambda_{2 j-1}-2 \mu_{j},
$$

which is continuous on $T$. The results of Sect. 5 include that under translation by $\xi$, every potential $q \in \mathscr{T}$ is given as the evaluation of $Q$ along a continuous path $\varphi(\xi)$ on the torus $T$. All this reminds one of the periodic case; however, now the orbit is not necessarily closed. Section 5 also includes a discussion of the closure of this orbit on $T$, a description of the dynamics of the orbit, and a derivation of the analogue of the Dubrovin vector field for $\boldsymbol{\mu}$ in terms of the Green's function for (1.1). In particular, it is shown that the vector field is autonomous.

The analysis for an existence theorem for integral curves of the Dubrovin vector field involves consideration of the geometry of the spectrum. In Sect. 6, a subclass of potentials in $\mathscr{T}$ is considered, for which the vector field admits a global $C^{1}$ bound. Existence, uniqueness, and continuous dependence of solutions are immediate consequences. The criterion specifying this subclass is in terms of the torus $T$, or equivalently in terms of the geometry of the spectrum $\sigma(L(q)) \subset \mathbb{R}$. For this subclass the mapping from $q(x+\xi)$ to $T$ is shown to extend continuously to the hull $\mathscr{H}(q)$ of $q$ :

$$
\mathscr{H}(q)=\overline{\{q(x+\xi) ; \xi \in \mathbb{R}\}},
$$

where closure is taken with respect to the topology of uniform convergence on compact sets. One conclusion is that the hull is compact. The $C^{1}$ estimate is given in Theorem 6.2. Specific estimates give that many potentials admit this global $C^{1}$ bound, including ones with Cantor spectra such as the examples of Moser [16] and Avron and Simon [1]. Global $C^{1}$ bounds on the Dubrovin vector field have been derived in the periodic case by Trubowitz [18].

There is a connection between this discussion of the trace formula and the study of Schrödinger operators with ergodic potentials. These are operators of form (1.1), whose potential $q(x)$ possesses some form of recurrence properties; the definition is given precisely in Sect. 4. Via a result of Kotani [9], an ergodic potential for which the Lyapounov exponent vanishes almost everywhere on the spectrum belongs to the reflectionless class $\mathscr{T}$, and thus is recoverable by formula (1.3) from a continuous orbit on a compact torus. Since the Lyapunov exponent vanishes on a dense subset of the absolutely continuous spectrum, this suggests the following appealing conjecture: if $q(x)$ is ergodic and $\sigma(L(q))=\sigma_{\mathrm{ac}}(L(q))$, then in fact, $q(x)$ is an almost periodic function. If this were true, the following alternative holds for ergodic Schrödinger operators: if the singular spectrum is empty, $\sigma_{\text {sing }}(L(q))=\sigma_{\mathrm{pp}}(L(q)) \cup \sigma_{\mathrm{sc}}(L(q))=\phi$, then the potential $q(x)$ is almost periodic. The results of Sects. 5 and 6 prove the following weaker conclusion: if $q(x)$ is ergodic and the Lyapounov exponent vanishes almost everywhere on the 
spectrum, then (i) the hull $\mathscr{H}(q)$ is compact, or else (ii) the spectrum $\sigma(L(q))$ is a wild set, not satisfying the hypotheses of Theorem 6.2. Roughly, such a wild set must possess accumulation points of endpoints of spectral gaps, with the sizes of converging gaps quite large compared to their relative distances apart. By contrast, standard Cantor sets of large relative Lebesgue measure are not wild sets.

This article has not addressed several natural questions. First, the Dubrovin vector field on $T$ has formally the structure of a completely integrable Hamiltonian system. For $q(x)$ within a restricted subclass of $\mathscr{T}$, Levitan has considered limits of the Jacobi inverse problem, integrated the motion, and showed that in fact $q(x)$ is almost periodic, a uniform limit of lacunary (finite spectral band) potentials. In Kotani and Krishna [10], it is remarked that Levitan's subclass includes spectral sets in which certain finite limit points of the set $\left\{\lambda_{j}\right\}_{j=0}^{\infty}$ are admissible. Their conditions, however, exclude any Cantor spectrum. Levitan's analysis has not been carried out for a broader subclass of $\mathscr{T}$. Secondly, the torus $T$ should represent the reflectionless spectral class of the operator $L(q)$, and the $\mathrm{KdV}$ and higher $\mathrm{KdV}$ vector fields should be identified and their initial value problems solved. This work has been done, including $C^{1}$ estimates for the higher $\mathrm{KdV}$ vector fields; it will appear in a subsequent publication.

\section{The First Half of the Trace Formula}

The approach that gives a proof of the trace formula in our general setting is to compute the trace of the difference of two heat kernels. In this section we evaluate this quantity in the first of two different ways. For $q(x)$ a bounded continuous function consider the Sturm-Liouville problem on the line

$$
L(q) \psi=\left(-\frac{d^{2}}{d x^{2}}+q(x)\right) \psi=\lambda \psi \quad-\infty<x<+\infty,
$$

and the one on the line, with Dirichlet conditions imposed at $x=0$.

$$
\begin{aligned}
L_{D}(q) \psi & =\left(-\frac{d^{2}}{d x^{2}}+q(x)\right)=\lambda \psi \quad-\infty<x<0 \text { and } 0<x<+\infty \\
\psi(0) & =0 .
\end{aligned}
$$

The integral kernels $H$ and $H_{D}$ of the solution operators for the two heat equations

$$
\frac{\partial}{\partial t} u+\frac{1}{2} L(q) u=0 \quad-\infty<x<+\infty
$$

and

$$
\frac{\partial}{\partial t} u+\frac{1}{2} L_{D}(q) u=0 \quad-\infty<x<0 \text { and } 0<x<+\infty
$$

are conveniently expressed using the Feynman-Kac formula,

$$
H(x, y ; t)=E_{x}\left(\exp \left(-\frac{1}{2} \int_{0}^{t} q(\beta(s)) d s\right) \delta_{y}(\beta(t))\right)
$$


and

$$
H_{D}(x, y ; t)=E_{x}\left(\exp \left(-\frac{1}{2} \int_{0}^{t} q(\beta(s)) d s\right) \chi_{\left\{t<T_{0}(\beta)\right\}} \delta_{y}(\beta(t))\right) .
$$

The path integral is with respect to the classical Brownian motion process on $\mathbb{R}$, with $\beta(0)=x, \delta_{y}$ is the Dirac measure at $y \in \mathbb{R}$, and $T_{0}(\beta)$ is the first hitting time of zero for the path $\beta(t)$;

$$
T_{0}(\beta)=\inf \{t>0 ; \beta(t)=0\} .
$$

In the free case, $q(x) \equiv 0$, the heat kernels (2.5) (2.6) are given explicitly by the method of images,

$$
H^{0}(x, y ; t)=\frac{1}{\sqrt{2 \pi t}} e^{-\frac{(x-y)^{2}}{2 t}}
$$

and

$$
H_{D}^{0}(x, y ; t)= \begin{cases}\frac{1}{\sqrt{2 \pi t}}\left(e^{-\frac{(x-y)^{2}}{2 t}}-e^{\left.-\frac{(x+y)^{2}}{2 t}\right)}\right. & \text { if } x, y>0 \text { or } x, y<0 \\ 0 & \text { if } y<0<x \text { or } x<0<y .\end{cases}
$$

On the diagonal $\{x=y\}$

$$
H^{0}(x, x ; t)-H_{D}^{0}(x, x ; t)=\frac{1}{\sqrt{2 \pi t}} e^{-\frac{2 x^{2}}{t}},
$$

and we have that

$$
\int_{-\infty}^{\infty} H^{0}(x, x ; t)-H_{D}^{0}(x, x ; t) d x=\frac{1}{2}
$$

Theorem 2.1. Let $q(x)$ be a bounded measurable function on $R$ which is continuous at $x=0$. Then

$$
\lim _{t \rightarrow 0^{+}} \frac{d}{d t} \int_{-\infty}^{\infty} H(x, x ; t)-H_{D}(x, x ; t) d x=-\frac{q(0)}{4} .
$$

Proof. Consider the quantity

$$
\begin{aligned}
H(x, x ; t)-H_{D}(x, x ; t) & =E_{x}\left(\exp \left(-\frac{1}{2} \int_{0}^{t} q(\beta(s) d s)\left(1-\chi_{\left\{t<T_{0}\right\}}\right) \delta_{x}(\beta(t))\right)\right. \\
& =\sum_{j=0}^{\infty} \frac{1}{j !} E_{x}\left(\left(-\frac{1}{2} \int_{0}^{t} q(\beta(s)) d s\right)^{j}\left(1-\chi_{\left\{t<T_{0}\right\}}\right) \delta_{x}(\beta(t))\right) .
\end{aligned}
$$

Since $\|q\|_{\infty}<\infty$, the series is convergent. The term $j=0$ of the series is easily evaluated,

$$
\int_{-\infty}^{\infty} E_{x}\left(\left(1-\chi_{\left\{t<T_{0}\right\}}\right) \delta_{x}(\beta(t))\right) d x=\int_{-\infty}^{\infty} H^{0}(x, x ; t)-H_{D}^{0}(x, x ; t) d x=\frac{1}{2} .
$$


For $j \geqq 2$ we have

$$
\begin{aligned}
& \left|\sum_{j=2}^{\infty} \frac{1}{j !} E_{x}\left(\left(-\frac{1}{2} \int_{0}^{t} q d s\right)^{j}\left(1-\chi_{\left\{t<T_{0}\right\}}\right) \delta_{x}(\beta(t))\right)\right| \\
& \quad \leqq \sum_{j=2}^{\infty} \frac{1}{j !}\left(\frac{t}{2}\|q\|_{L \infty}\right)^{j} E_{x}\left(\left(1-\chi_{\left\{t<T_{0}\right\}}\right) \delta_{x}(\beta(t))\right)
\end{aligned}
$$

which for $t<1$ is bounded by $C t^{2} e^{-2 x^{2} / t} / \sqrt{2 \pi t}$. Thus as $t \rightarrow 0^{+}$,

$$
\int_{-\infty}^{\infty} \sum_{j=2}^{\infty} \frac{1}{j !} E_{x}\left(\left(-\frac{1}{2} \int_{0}^{t} q d s\right)^{j}\left(1-\chi_{\left\{t<T_{0}\right\}}\right) \delta_{x}(\beta(t))\right) d x=O\left(t^{2}\right) .
$$

It remains to evaluate the term $j=1$,

$$
\begin{gathered}
\int_{-\infty}^{\infty} E_{x}\left(\left(-\frac{1}{2} \int_{0}^{t} q(\beta(s)) d s\right)\left(1-\chi_{\left\{t<T_{0}\right\}}\right) \delta_{x}(\beta(t))\right) d x \\
=-\frac{1}{2} \int_{0}^{t} \int_{-\infty}^{\infty} E_{x}\left(q(\beta(s))\left(1-\chi_{\left\{t<T_{0}\right\}}\right) \delta_{x}(\beta(t))\right) d x d s \\
=-\frac{1}{2} \int_{0}^{t} \int_{-\infty}^{\infty} \int_{-\infty}^{\infty} H^{0}(x, y ; s) q(y) H^{0}(y, x ; t-s) \\
-H_{D}^{0}(x, y ; s) q(y) H_{D}^{0}(y, x ; t-s) d y d s .
\end{gathered}
$$

Using the semigroup property of the heat operator and interchanging integrations:

$$
\begin{aligned}
= & -\frac{1}{2} \int_{0}^{t} \int_{-\infty}^{\infty} q(y) \int_{\infty}^{\infty} H^{0}(y, x ; t-s) H^{0}(x, y ; s) \\
& -H_{D}^{0}(y, x ; t-s) H^{0}(x, y ; s) d x d y d s \\
= & -\frac{1}{2} \int_{0}^{t} \int_{-\infty}^{\infty} q(y)\left(H^{0}(y, y ; t)-H_{D}^{0}(y, y ; t)\right) d y d s \\
= & -\frac{t}{2} \int_{-\infty}^{\infty} q(y) \frac{1}{\sqrt{2 \pi t}} e^{-\frac{2 y^{2}}{t}} d y
\end{aligned}
$$

It is now clear that $\lim _{t \rightarrow 0^{+}} \int_{-\infty}^{\infty} H(x, x ; t)-H_{D}(x, x ; t) d x=1 / 2$, and that

$$
\begin{aligned}
& \lim _{t \rightarrow 0^{+}} \frac{d}{d t} \int_{-\infty}^{\infty} H(x, x ; t)-H_{D}(x, x ; t) d x \\
& \quad=\lim _{t \rightarrow 0^{+}} \frac{1}{t}\left(\int_{-\infty}^{\infty} H(x, x ; t)-H_{D}(x, x ; t) d x-\int_{-\infty}^{\infty} H^{0}(x, x ; t)-H_{D}^{0}(x, x ; t) d x\right) \\
& \quad=\lim _{t \rightarrow 0^{+}}-\frac{1}{2} \int_{-\infty}^{\infty} q(y) \frac{1}{\sqrt{2 \pi t}} e^{-\frac{2 y^{2}}{t}} d y \\
& \quad=-\frac{q(0)}{4} .
\end{aligned}
$$

This completes the proof. 
It will be whown in Sect. 3 that formula (2.8) has the interpretation of the trace of the difference of $L(q)$ and $L_{D}(q)$. If the function $q(x)$ has better differentiability properties at $x=0$ then we may derive the trace of the difference of higher powers of the operators $L(q)$ and $L_{D}(q)$. In the periodic case these traces are discussed in [13]. In our present setting the first $1 / 2$ of these higher trace formulae are obtained.

Theorem 2.2. Suppose that $q(x)$ is bounded, and that all derivatives up to order $r$ are continuous at $x=0$. For $0 \leqq \alpha \leqq[r / 2]+1$,

$$
\lim _{t \rightarrow 0^{+}}\left(\frac{d}{d t}\right)^{\alpha} \int_{-\infty}^{\infty} H(x, x ; t)-H_{D}(x, x ; t) d x=P_{\alpha}\left(q(0), \ldots, q^{(2 \alpha-2)}(0)\right),
$$

where $P_{\alpha}$ are universal polynomials in $q(0), \ldots q^{(2 \alpha-2)}(0)$.

Proof. One uses the same strategy for higher derivatives that is used for the estimation and evaluation of terms for $\alpha=0,1$. The expansion of the exponential in the Feynman-Kac formula gives both a computation of $P_{\alpha}$ and an estimate of error terms. Only integral powers of $t$ will appear in the asymptotic expansion of $\int_{-\infty}^{\infty} H(x, x ; t)-H_{D}(x, x ; t) d x$ as $t-0^{+}$; this is expected because of its interpretation as the formal quantity $\operatorname{tr}\left(e^{-t L(q)}-e^{-t L_{D}(q)}\right)$.

Consider the expression (2.9). The $j^{\text {th }}$ term in the expansion of the exponential is

$$
\begin{aligned}
& E_{x}\left(\left(\int_{0}^{t} q(\beta(s)) d s\right)^{j}\left(1-\chi_{\left\{t<T_{0}\right\}}\right) \delta_{x}(\beta(t))\right) \\
& =\int_{0}^{t} j \times \int_{0}^{t} E_{x}\left(\prod_{l=1}^{j} q\left(\beta\left(s_{l}\right)\right)\left(1-\chi_{\left\{t<T_{0}\right\}}\right) \delta_{x}(\beta(t))\right) d s_{1} \ldots d s_{j} \\
& \quad=r(j) \int_{0}^{t} \int_{0}^{s_{j}-1} \cdots \int_{0}^{s_{2}} E_{x}\left(\prod_{l=1}^{j} q\left(\beta\left(s_{l}\right)\right)\left(1-\chi_{\left\{t<T_{0}\right\}}\right\} \delta_{x}(\beta(t))\right) d s_{1} \ldots d s_{j},
\end{aligned}
$$

where $r(j)$ is the number of rearrangement of the set $\left\{s_{1}, \ldots, s_{j}\right\}$. If $q(x)=\sum_{l=0}^{r} q^{(l)}(0) x^{l} / l !+o\left(|x|^{r}\right)$ as $x \rightarrow 0$, for small $t$, we use the Taylor expansion of the product $\prod_{l=1}^{j} q\left(\beta\left(s_{l}\right)\right)=\sum_{n \leqq r} \sum_{|\alpha|=n} \prod_{l=1}^{j} q^{\left(\alpha_{l}\right)}(0) \beta\left(s_{l}\right)^{\alpha_{l}}+e_{r}(\beta)$, where $e_{r}(\beta)=o\left(\left|\beta\left(s_{1}\right)\right|^{2}+\ldots+\left|\beta\left(s_{j}\right)\right|^{2}\right)^{r / 2}$ controls the error term. The resulting expression is

$$
\begin{aligned}
& r(j) \sum_{n \leqq r} \sum_{|\alpha|=n} \frac{1}{\alpha !} \prod_{l=1}^{j} q^{\left(\alpha_{l}\right)}(0) \int_{0}^{t} \cdots \int_{0}^{s_{2}} d s_{1} \ldots d s_{j} \\
& \quad E_{x}\left(\prod_{l=1}^{j} \beta^{\alpha_{l}}\left(s_{l}\right)\left(1-\chi_{\left\{t<T_{0}\right\}}\right) \delta_{x}(\beta(t))\right) \\
& \quad+E_{x}\left(\int_{0}^{t} \cdots \int_{0}^{t} e_{r}(\beta) d s_{1} \ldots d s_{j}\left(1-\chi_{\left\{t<T_{0}\right\}}\right\} \delta_{x}(\beta(t))\right) .
\end{aligned}
$$


The coefficients of the universal polynomials come from the integrals

$$
\int_{0}^{t} \cdots \int_{0}^{s_{2}} d s_{1} \ldots d s_{j} \int_{-\infty}^{\infty} d x E_{x}\left(\prod_{l=1}^{j} \beta^{\alpha_{l}}\left(s_{l}\right)\left(1-\chi_{\left\{\mathrm{t}<\mathrm{T}_{0}\right\}}\right) \delta_{x}(\beta(t))\right)=\sum_{l=1}^{\left[\frac{l}{2}\right]+j} a_{\alpha l} t^{l} .
$$

An explicit computation shows that if $\sum_{l=1}^{j} \alpha_{l}$ is odd then the integration in $x$ vanishes, hence only integral powers of $t$ appear in (2.12). The error term in (2.11) can also be shown to contribute only higher order behavior in $t$ as $t \rightarrow 0^{+}$. Computing the constants $a_{\alpha l}$ and ordering the resulting powers of $t$ up to $[r / 2]+1$ (which in practive may be tedious), the result follows.

These two results are adaptations of the method used in Deift and Trubowitz [4] and Douady [5]; they were worked out for me in this setting by V. Papanicolaou.

\section{The Second Half of the Trace Formula}

As the first half of the trace formula is obtained from perturbation theory for the heat kernel, the second half must concern the spectra of the operators $L(q)$ and $L_{D}(\mathrm{q})$. In this section we derive formulae in terms of the Green's function $g(x, y ; \lambda)$, its values on the diagonal $y=x$, and their limits on the real axis as $\operatorname{im} \lambda \rightarrow 0^{+}$. We have taken $q(x) \in C(\mathbb{R})$, bounded continuous functions on the line. It is well known that the resulting operators $L(q)$ and $L_{D}(q)$ are Weyl limit point case at $x \rightarrow \pm \infty$. Consider a fundamental solution matrix for Eq. (2.1),

$$
\Phi(x, \lambda)=\left(\begin{array}{ll}
\varphi_{1}(x, \lambda) & \varphi_{2}(x, \lambda) \\
\frac{d}{d x} \varphi_{1}(x, \lambda) & \frac{d}{d x} \varphi_{2}(x, \lambda)
\end{array}\right), \quad \Phi(0, \lambda)=I .
$$

For $\lambda$ in the upper (or lower) half plane $\mathbb{C}^{ \pm}$one constructs the limit points $m_{ \pm}(\lambda)$ and the stable and unstable solutions

$$
f_{ \pm}(x, \lambda)=\varphi_{1}(x, \lambda)+m_{ \pm}(\lambda) \varphi_{2}(x, \lambda) \in L^{2}\left(\mathbb{R}^{ \pm}\right) .
$$

Since the problem is real, $m_{ \pm}(\bar{\lambda})=\bar{m}_{ \pm}(\lambda)$, and $f_{ \pm}(x, \bar{\lambda})=\overline{f_{ \pm}}(x, \lambda)$. Computing the Wronskian at $x=0,\left[f_{+}, f_{-}\right]=m_{-}(\bar{\lambda})-m_{+}(\lambda)$, we obtain the expression for the Green's function for problem (2.1);

$$
g(x, y ; \lambda)=\frac{f_{+}(x, \lambda) f_{-}(y, \lambda)}{\left(m_{-}-m_{+}\right)(\lambda)}, \quad y \leqq x .
$$

The trace formula involves a comparison of this full line Green's function with the one for the half line problem (2.2);

$$
g_{D}(x, y ; \lambda)=\left\{\begin{array}{cc}
\varphi_{2}(y, \lambda) f_{+}(x, \lambda) & 0 \leqq y \leqq x \\
0 & y \leqq 0 \leqq x \\
-f_{-}(y, \lambda) \varphi_{2}(x, \lambda) & y \leqq x \leqq 0
\end{array}\right.
$$

The elementary properties of these functions are given in this lemma. 
Lemma 3.1. The Weyl $m$-functions $m_{ \pm}(\lambda)$ and the diagonal of the Green's function $g(x, x ; \lambda)$ are analytic in $\mathbb{C}^{ \pm}$. For $\operatorname{im} \lambda>0$,

$$
\operatorname{im} m_{+}(\lambda)>0, \quad \operatorname{im} m_{-}(\lambda)<0, \quad \operatorname{im} g(x, x ; \lambda)>0 .
$$

Analytic functions of $\lambda$ which satisfy this property of mapping the upper (lower) half plane to itself are called Herglotz; they play a central role in the spectral theory of Sturm-Liouville problems. The lemma states that $m_{+},-m_{-}$ and $g(x, x ; \lambda)$ are Herglotz.

Proof. First notice that $g(0,0 ; \lambda)=\left(m_{-}-m_{+}\right)^{-1}(\lambda)$; therefore the result for $g$ will follow from that for the Weyl $m$-functions. For the Weyl functions it is a computation that

$$
\frac{d}{d x}\left[f_{ \pm}, \overline{f_{ \pm}}\right]=f_{ \pm} \frac{d^{2}}{d x^{2}} \overline{f_{ \pm}}-\frac{d^{2}}{d x^{2}} f_{ \pm} \overline{f_{ \pm}}=2 i \mathrm{im} \lambda\left|f_{ \pm}\right|^{2}
$$

Thus

$$
\operatorname{im} m_{ \pm}(\lambda)=\frac{i}{2}\left[f_{ \pm}, \overline{f_{ \pm}}\right]=\operatorname{im} \lambda \int_{0}^{ \pm \infty}\left|f_{ \pm}\right|^{2} d x,
$$

from which the result follows.

The second elementary result that we use is that the Weyl $m$-functions and thus the diagonal of the Green's function are continuous as functions of the potential $q$, considered in the topology of uniform convergence on compact sets.

Lemma 3.2. If $q_{n}(x)$ converges to $q(x)$ uniformly on compact sets, then $m_{+}^{n}(\lambda)$ converge to $m_{ \pm}(\lambda)$. This convergence is uniform in $\lambda$, for $\lambda$ in compact sets of $\mathbb{C}^{ \pm}$.

The proof of this lemma has appeared in several settings. See, for example, Johnson and Moser [8], or Craig [3], in which the nesting property of the Weyl circles are used.

The spectrum $\sigma(L(q))$ of $L(q)$ may be a quite complicated set on the real axis. There may be isolated points, bands, Cantor sets or other phenomena. It is bounded below, however, and the complement of the spectrum consists of at most countable many disjoint open intervals. We denoted the bottom of the spectrum $\lambda_{0}$, and enumerate the spectral gaps $I_{j}=\left(\lambda_{2 j-1}, \lambda_{2 j}\right)$,

$$
\mathbb{R}-\sigma(L(q))=\left(-\infty, \lambda_{0}\right) \cup \bigcup_{j=1}^{\infty} I_{j} .
$$

The principal concern of this section is to obtain a trace formula in the cases in which the potential can be described by quantities outside the spectrum. The quantities of interest will be the collection of gap endpoints $\left\{\lambda_{j}\right\}_{j=0}^{\infty}$ and the zeros of the diagonal of the Green's function in each gap, which are traditionally denoted $\left\{\mu_{j}\right\}_{j=1}^{\infty}$.

Lemma 3.3. The function $g(0,0 ; \lambda)$ is real and increasing for $\lambda \in I_{j}$. Thus there is at most one zero $\lambda=\mu_{j}$ in each gap. 
Proof. $I_{j}$ 's are regions of analyticity of $g(0,0 ; \lambda)$, and furthermore $g(0,0 ; \lambda)=\overline{g(0,0 ; \lambda)}$, so that within the gap the diagonal of the Green's function is smooth and real valued. We compute

$$
\frac{d}{d \lambda} g(0,0 ; \lambda)=\int_{-\infty}^{\infty} g^{2}(0, x ; \lambda) d x>0,
$$

hence $g$ is strictly increasing in $\lambda$ in each gap, and any zero must be unique.

If $g(0,0 ; \lambda)$ vanishes in the open interval $I_{j}$, we denote that point $\mu_{j}$. It can, however, occur that $g$ will have entirely one sign throughout $I_{j}$. If $g<0$ in $I_{j}$ set $\mu_{j}=\lambda_{2 j}$, the right endpoint, while if $g>0$, set $\mu_{j}=\lambda_{2 j-1}$, the left endpoint. A zero of $g(0,0 ; \lambda)$ in $I_{j}$ corresponds to a pole of either one of $m_{ \pm}(\lambda)$, and therefore to an eigenvalue in the Dirichlet spectrum for either the right or the left half line problems (2.2).

- Using the spectral representation of the solution operators to the two heat equations (2.3) and (2.4), we obtain the following formulae. Let $\Gamma$ be a contour around the spectrum of both $L(q)$ and $L_{D}(q)$; for example,

$$
\Gamma=\left\{\lambda_{0}-\delta+i \eta ; \quad \varepsilon \leqq g \leqq \varepsilon\right\} \cup\left\{(\xi \pm i \varepsilon) ; \quad \lambda_{0}-\delta \leqq \xi \leqq+\infty\right\} .
$$

Lemma 3.4. The trace of the difference of the two heat kernels satisfies

$$
\int_{-\infty}^{\infty} H(x, x ; t)-H_{D}(x, x ; t) d x=\frac{1}{2 \pi i} \int_{\Gamma} e^{-\lambda t / 2} \frac{d}{d \lambda}\left(\log g(0,0 ; \lambda)-\frac{i \pi}{2}\right) d \lambda .
$$

A similar expression is obtained if we consider the difference $f(L(q))-f\left(L_{D}(q)\right)$ of any function $f$ in the bounded spectral calculus for which $f^{\prime}$ is integrable on the ray $\left[\lambda_{0},+\infty\right)$; the heat kernel has of course $f(\lambda)=e^{-\lambda t / 2}$.

Proof. This formula follows from a computation involving the two Green's functions. The left-hand side of (3.4) can be written in terms of a contour integral over $\Gamma$;

$$
\frac{1}{2 \pi i} \int_{-\infty}^{\infty} \int_{\Gamma} e^{-\lambda t / 2}\left(g(x, x ; \lambda)-g_{D}(x, x ; \lambda)\right) d \lambda d x .
$$

Compute the difference of the Green's functions;

$$
\begin{aligned}
g(x, x ; \lambda)-g_{D}(x, x ; \lambda) & =\frac{f_{+}(x, \lambda) f_{-}(x, \lambda)}{m_{-}(\lambda)-m_{+}(\lambda)}-\left\{\begin{array}{rr}
f_{+}(x, \lambda) \varphi_{2}(x, \lambda) & x \geqq 0 \\
-f_{-}(x, \lambda) \varphi_{2}(x, \lambda) & x \leqq 0
\end{array}\right. \\
& = \begin{cases}f_{+}\left(f_{-}-\left(m_{-}-m_{+}\right) \varphi_{2}\right) /\left(m_{-}-m_{+}\right) & x \geqq 0 \\
f_{-}\left(f_{+}+\left(m_{-}-m_{+}\right) \varphi_{2}\right) /\left(m_{-}-m_{+}\right) & x \leqq 0\end{cases} \\
& = \begin{cases}f_{+}^{2}(x, \lambda) /\left(m_{-}-m_{+}\right) & x \geqq 0 \\
f_{-}^{2}(x, \lambda) /\left(m_{-}-m_{+}\right) & x \leqq 0\end{cases}
\end{aligned}
$$

By the resolvant formula we recognize that $d / d \lambda R(\lambda)=R^{2}(\lambda)$, hence in terms of integral kernels $d g(0,0 ; \lambda) / d \lambda=\int_{-\infty}^{\infty} g^{2}(x, 0 ; \lambda) d x$. Thus from (3.2) and (3.6):

$$
\int_{-\infty}^{\infty} g(x, x ; \lambda)-g_{D}(x, x ; \lambda) d x=\left(m_{-}-m_{+}\right)(\lambda) \int_{-\infty}^{\infty} g^{2}(x, 0 ; \lambda) d x=\frac{d}{d \lambda} \log g(0,0 ; \lambda) \text {. }
$$

With a change of constant, this gives formula (3.4). 
Formula (3.4) is generally valid, and in a sense is comparable to the trace formulae of Deift and Trubowitz, or McKean, can Moerbeke, and Trubowitz. In this paper I want to discuss a special class of potentials $q(x)$ for which the righthand side can be evaluated more explicitly at $t \rightarrow 0^{+}$. Let

$$
\begin{aligned}
\mathscr{T}= & \left\{q \in C(\mathbb{R}) ; \lim _{\varepsilon \rightarrow 0^{+}} \operatorname{re} g(x, x ; \lambda+i \varepsilon)=0\right. \\
& \text { for all } x \in \mathbb{R} \text {, for almost all } \lambda \in \sigma(L(q))\} .
\end{aligned}
$$

We will call this class of $q$ the "reflectionless" potentials. From Lemmas 3.1 and 3.2 it is clear that

$$
\lim _{\varepsilon \rightarrow 0} \operatorname{im} g(x, x ; \lambda+i \varepsilon)=0
$$

for all $\lambda \in \mathbb{R}-\sigma(L(q))$, and that the argument of $g(x, x ; \lambda)$ in $\mathbb{C}^{+}$is bounded between 0 and $\pi$.

This class of reflectionless potentials includes many of the well-studied classes of spectral and inverse spectral theory. Among these are (i) all continuous periodic potentials [14], (ii) the reflectionless potentials of Bargmann [2], (iii) finite gap potentials studied by Dubrovin, Matveev and Novikov [6], (iv) potentials constructed by Moser and Trubowitz that are related to orbits of the constrained harmonic oscilator problem of C. Neumann [15], and all examples known to the author of constructions of nowhere dense spectra with limit periodic potentials. Another case that is of great interest is the class of ergodic potentials which posess entirely absolutely continuous spectrum. The definition of an ergodic potential and the theorem that ergodic plus absolutely continuous spectrum implies reflectionless will be given in Sect. 4; this result follows from the work of Kotani [9].

Using (3.4) we obtain the following general trace formula that can be used as an inversion theorem for reflectionless potentials:

Theorem 3.6. Let $q \in \mathscr{T}$; then

$$
\int_{-\infty}^{\infty} H(x, x ; t)-H_{D}(x, x ; t) d x=\frac{1}{2} e^{\frac{-\lambda_{0} t}{2}}+\frac{1}{2} \sum_{j=1}^{\infty}\left(e^{\frac{-\lambda_{2 j} t}{2}}+e^{\frac{-\lambda_{2 j}-1 t}{2}}-2 e^{\frac{-\mu_{j} t}{2}}\right) .
$$

Thus if $\sum_{j=1}^{\infty}\left|I_{j}\right|<\infty$, the second half of the trace formula holds;

$$
q(0)=-\lambda_{0}+\sum_{j=1}^{\infty}\left(\lambda_{2 j}+\lambda_{2 j-1}-2 \mu_{j}\right) .
$$

Denote the midpoint of the gap $I_{j}$ by $\xi_{j}=\left(\lambda_{2 j}+\lambda_{2 j-1}\right) / 2$. If the higher moments are finite

$$
\sum_{j=1}^{\infty}\left|I_{j}\right| \xi_{j}^{[r / 2]}<\infty
$$

and the coefficient $q(x) \in C^{r}(\mathbb{R})$, then the $\gamma$ trace formulae hold

$$
P_{\gamma}\left(q(0), \ldots, q^{(2 \gamma-2)}(0)\right)=\left(-\frac{1}{2}\right)^{\gamma}\left(\lambda_{0}^{\gamma}+\sum_{j=1}^{\infty} \lambda_{2 j}^{\gamma}+\lambda_{2 j-1}^{\gamma}-2 \mu_{j}^{\gamma}\right)
$$

for $0 \leqq \gamma \leqq[r / 2]+1$. 
Proof. We of course want to take the limit as $\varepsilon \rightarrow 0^{+}$of the contour integral in (3.4). In general there will be a contribution to the result from the continuous spectrum, a case that we do not consider in this article. The reflectionless condition is precisely the one for which this contribution is zero. From (3.4),

$$
\begin{aligned}
\frac{1}{2 \pi i} \int_{\Gamma} e^{-\lambda t / 2} \frac{d}{d \lambda}\left(\log g(0,0 ; \lambda)-\frac{i \pi}{2}\right) d \lambda \\
=\frac{1}{2 \pi i} \int_{\lambda_{0}-\delta-i \varepsilon}^{\lambda_{0}-\delta+i \varepsilon} e^{-\lambda t / 2} \frac{d}{d \lambda}\left(\log g(0,0 ; \lambda)-\frac{i \pi}{2}\right) d \lambda \\
\quad-\frac{1}{\pi} \int_{\lambda_{0}-\delta+i \varepsilon}^{\infty+i \varepsilon} \operatorname{im}\left(e^{-\lambda t / 2} \frac{d}{d \lambda}\left(\log g(0,0 ; \lambda)-\frac{i \pi}{2}\right)\right) d \lambda .
\end{aligned}
$$

The first term will vanish as $\varepsilon \rightarrow 0$ while in the second we integrate by parts,

$$
\begin{aligned}
& \frac{1}{\pi} \int_{\lambda_{0}-\delta}^{+\infty} \frac{t}{2} \operatorname{im}\left(e^{-(\xi+i \varepsilon) t / 2}\left(\log g(0,0 ; \xi+i \varepsilon)-\frac{i \pi}{2}\right)\right) d \xi \\
& \quad-\frac{1}{\pi} \operatorname{im}\left(e^{-\left(\lambda_{0}-\delta+i \varepsilon\right) t / 2}\left(\log g\left(0,0 ; \lambda_{0}-\delta+i \varepsilon\right)-\frac{i \pi}{2}\right)\right) .
\end{aligned}
$$

One takes the limit $\varepsilon \rightarrow 0$ using the dominated convergence theorem; we obtain

$$
\frac{1}{2} e^{-\left(\lambda_{0}-\delta\right) t / 2}+\frac{1}{\pi} \int_{\lambda_{0}-\delta}^{+\infty} \frac{t}{2} e^{-\xi t / 2}\left(\operatorname{im} \log g(0,0 ; \xi+i 0)-\frac{\pi}{2}\right) d \xi
$$

In the reflectionless case the limit of the function im $\log g(0,0 ; \lambda+i 0)-\pi / 2=0$ for almost every $\lambda \in \sigma(L(q))$. Since $g(0,0 ; \lambda)$ is Herglotz, for $\lambda \notin \sigma(L(q)), \lambda \neq \mu_{j}$ for some $j$, there are two possibilities;

$$
\left\{\begin{array}{ll}
\text { im } \log g(0,0 ; \lambda+i 0)-\frac{\pi}{2}=-\frac{\pi}{2} & \text { for } g(0,0 ; \lambda+i 0)>0 \\
\operatorname{im} \log g(0,0 ; \lambda+i 0)-\frac{\pi}{2}=\frac{\pi}{2} & \text { for } g(0,0 ; \lambda+i 0)<0
\end{array} .\right.
$$

Taking $\delta \rightarrow 0$ and integrating over each spectral gap,

$$
\begin{array}{r}
\frac{1}{2} e^{\frac{-\lambda_{0} t}{2}}+\frac{t}{2 \pi} \int_{\lambda_{0}}^{+\infty} e^{\frac{-\lambda t}{2}}\left(\sum_{j=1}^{\infty} \frac{\pi}{2} \chi_{\left[\lambda_{2 j}-1, \mu_{j}\right]}-\frac{\pi}{2} \chi_{\left[\mu_{,}, \lambda_{2 j}\right]}\right) d \lambda \\
=\frac{1}{2}\left(e^{\frac{-\lambda_{0} t}{2}}+\sum_{j=1}^{\infty}\left(e^{\frac{-\lambda_{2 j} t}{2}}+e^{\frac{-\lambda_{2 j-1} t}{2}}-2 e^{\frac{-\mu_{j} t}{2}}\right)\right),
\end{array}
$$

which is formula (3.8). In case the gap lengths $\left|I_{j}\right|=\left(\lambda_{2 j}-\lambda_{2 j-1}\right) / 2$ are summable, the time derivative of (3.8) has a finite limit as $t \rightarrow 0^{+}$, giving formula (3.9) in conjunction with Theorem 2.1 . Of course with more control on the asymptotic rate of decrease of gap widths, more derivatives of formula (3.8) will have limits as $t \rightarrow 0^{+}$. Sufficient control in terms of moments is stated in (3.10), which together with Theorem 2.2 proves (3.11). An alternative condition to (3.10) is the moment condition

$$
\int_{-\infty}^{\infty} \sum_{j=1}^{\infty} \chi_{\left[\lambda_{2 j-1}, \lambda_{2 j}\right]} \xi^{\left[\frac{i}{2}\right]} d \xi<\infty
$$


If a potential $q(x)$ has only finite differentiability properties at $x=0$, then for some $\gamma$ the limit $\lim _{t \rightarrow 0^{+}}(d / d t)^{\gamma} \int_{-\infty}^{-\infty} H(x, x ; t)-H_{D}(x, x ; t) d x$ diverges. This implies a converse; the moment estimates (3.10) and (3.14) cannot hold, and there must exist relatively many relatively large gaps as $\lambda \rightarrow+\infty$.

The first half of the trace formula holds independently of the summability of the gap lengths $\sum_{j=1}^{\infty}\left|I_{j}\right|<\infty$. It would be pleasing if the second half would be independent of this condition as well. In the periodic case these asymptotics of gap lengths are obtained as consequences of smoothness of the potential $q(x)$; in the general case we have not been able to prove similar results.

I would like to end this section with a justification of the term "reflectionless". When a potential $q(x)$ is given which presents a scattering situation, for example if $\int_{-\infty}^{\infty}|q(x)|\left(1-x^{2}\right) d x<\infty$, the spectral and inverse spectral problems are discussed in terms of the Jost functions. These are solutions of (2.1) satisfying

$$
\begin{array}{ll}
\psi_{+}(x, k) \sim e^{i k x} & \text { as } x \rightarrow+\infty, \\
\psi_{-}(x, k) \sim e^{-i k x} & \text { as } x \rightarrow-\infty
\end{array}
$$

for $k \in \mathbb{R}$ such that $k^{2}=\lambda$. Their asymptotic behavior at the other extreme of the $x$-axis is given in terms of transmission and reflection coefficients;

$$
\begin{aligned}
& \psi_{+}(x, k) \sim \frac{1}{T(k)} e^{i k x}+\frac{R_{+}(k)}{T(k)} e^{-i k x} \quad \text { as } x \rightarrow-\infty, \\
& \psi_{-}(x, k) \sim \frac{R_{-}(k)}{T(k)} e^{i k x}+\frac{1}{T(k)} e^{-i k x} \quad \text { as } x \rightarrow+\infty .
\end{aligned}
$$

From (3.15) it is clear that for $k>0, \psi_{ \pm}(x, k)$ represent the same solutions as the Weyl solutions $\lim _{\operatorname{im} \lambda \rightarrow 0^{+}} f_{ \pm}(x, \lambda), k^{2}=\lambda$, with however a different normalization at $x=0$. Computing Wronskians, we see a correspondence between Weyl $m$-functions and $R_{ \pm}(k)$ and $T(k)$;

$$
\begin{aligned}
& {\left[f_{+}, \overline{f_{+}}\right]=\left(\bar{m}_{+}-m_{+}\right)=\frac{-2 i k}{\left|\psi_{+}(0, k)\right|^{2}},} \\
& {\left[f_{-}, \overline{f_{-}}\right]=\left(\bar{m}_{-}-m_{-}\right)=\frac{2 i k}{\left|\psi_{-}(0, k)\right|^{2}},} \\
& {\left[f_{+}, f_{-}\right]=\left(m_{-}-m_{+}\right)=\frac{1}{\psi_{+}(0, k) \psi_{-}(0, k)} \frac{-2 i k}{T(k)},} \\
& {\left[f_{+}, \overline{f_{-}}\right]=\left(\bar{m}-m_{+}\right)=\frac{1}{\psi_{+}(0, k) \overline{\psi_{-}(0, k)}} \frac{2 i k}{T(k)} R_{+}(k) .}
\end{aligned}
$$

The other scattering theoretic identities are that

$$
\begin{gathered}
|T(k)|^{2}+\left|R_{ \pm}(k)\right|^{2}=1, \\
T(k) \bar{R}_{+}(k)+\bar{T}(k) R_{-}(k)=0 .
\end{gathered}
$$


For $\lambda=k^{2}$ such that $\operatorname{im} m_{ \pm}(\lambda) \neq 0$ it is clear that $R_{+}(k)=0$ corresponds to the condition that $\operatorname{re} g(0,0 ; \lambda)=\left(\operatorname{re} m_{-}(\lambda)-\operatorname{re} m_{+}(\lambda)\right) /\left|\left(m_{-}-m_{+}\right)(\lambda)\right|^{2}=0$.

\section{Ergodic Potentials}

A coefficient $q(x)$ in Eq. (2.1) is called an ergodic potential if it has certain properties of recurrence under translation of $x$. Potentials of this kind have been widely studied in the past decade, as they are taken to be reasonable models of quantum mechanical problems in the presence of some randomness or disorder. The setup we describe below applies in great generality, and the word ergodic will refer to a large class of functions $q(x)$ that include periodic and almost periodic potentials as well as the genuinely random potentials of the class considered by Goldsheid, Molchanov, and Pastur [7] and others. The spectrum, spectral decomposition, and many other functionals of $q$ can be shown to be constant almost everywhere with respect to an underlying probability measure, and thus can be defined as functionals of that measure.

Let the class of potentials $q(x)$ which we consider be uniformly bounded on $\mathbb{R}$; without loss of generality suppose that $-1 \leqq q(x) \leqq 1$. We take the unit ball $B_{1} \subseteq C(\mathbb{R})$ to be a probability space $\Omega$, endowed with the topology of uniform convergence on compact sets. There is a transformation of $\Omega$ corresponding to translation on the line; $\left(\varphi_{s} q\right)(x)=q(x+s)$, for $-\infty<s<+\infty$. A probability measure $P$ on $\Omega$ gives rise to a class of ergodic potentials if (i) $P$ is $\varphi_{s}$ invariant and (ii) $\varphi_{s}$ is ergodic with respect to $P$. In order to integrate functionals of $q \in \Omega$, the expectation value is defined by

$$
E_{P}(f(q))=\lim _{R \rightarrow \infty} \frac{1}{R} \int_{0}^{R} f\left(\varphi_{s}\right) d s,
$$

which by ergodicity is constant for $P$-almost every $q$. The support $\operatorname{supp} P$ of a measure $P$ on $\Omega$ is taken with respect to the topology of uniform convergence on compact sets of $\mathbb{R}$.

Lemma 4.1. (Pastur[17]). The spectral decomposition holds for P-almost every $q \in \Omega$. That is, there exist sets $\sigma(P)=\sigma_{\mathrm{ac}}(P) \cup \sigma_{\mathrm{sc}}(P) \cup \sigma_{\mathrm{pp}}(P) \subseteq \mathbb{R}$ such that for $P$-almost every $q(x)$;

$$
\begin{aligned}
\sigma(L(q)) & =\sigma(P), \\
\sigma_{\mathrm{ac}}(L(q)) & =\sigma_{\mathrm{ac}}(P), \\
\sigma_{\mathrm{sc}}(L(q)) & =\sigma_{\mathrm{sc}}(P), \\
\sigma_{\mathrm{pp}}(L(q)) & =\sigma_{\mathrm{pp}}(P) .
\end{aligned}
$$

The Floquet exponent $w(\lambda)$ for an ergodic potential is defined for $\operatorname{im} \lambda \neq 0$ by

$$
w(\lambda)=E_{P}\left(-\frac{1}{2 g(0,0 ; \lambda)}\right)
$$

it furthermore has almost everywhere nontangential limits on the real axis. The Lyapounov exponent is $\gamma(\lambda)=-\operatorname{re} w(\lambda)$. Previous sections of this article are concerned with the set of $\lambda \in \sigma(L(q))$ for which the zero reflection property holds; 
$\lim _{\epsilon \rightarrow \infty}$ re $g(x, x ; \lambda+i \varepsilon)=0$. In the ergodic case this can be stated in terms of the vanishing of the Lyapounov exponent.

Theorem 4.2 (Kotani [9]). Let $P$ be an ergodic probability measure on $\Omega$. For any $q \in \operatorname{supp} P$ let $A=\{\lambda \in \mathbb{R} ; \gamma(\lambda)=0\}$. For almost every $\lambda \in A$,

$$
m_{+}(\lambda+i 0)=\bar{m}_{-}(\lambda+i 0)=m_{-}(\lambda-i 0) .
$$

Furthermore, for every $x \in \mathbb{R}$, for almost every $\lambda \in A$,

$$
\operatorname{re} g(x, x ; \lambda+i 0)=0 \text {. }
$$

This is a very beautiful result that has appeared in Kotani [9], to which the reader is referred for a proof. We mention here, however, that the result follows from certain covariance properties of the Weyl functions $m_{ \pm}(\lambda)$ and their expectation values. As an immediate consequence we have:

Corollary 4.3. If $q \in \operatorname{supp} P$ is an ergodic potential such that meas $(\sigma(L(q))-A)=0$, then for all $x \in \mathbb{R}$, for almost all $\lambda \in \sigma(L(q))$,

$$
\lim _{\epsilon \rightarrow \infty} \operatorname{reg}(x, x ; \lambda+i \varepsilon)=0 .
$$

That is, an ergodic potential for which the Lyapounov exponent vanishes almost everywhere on the spectrum is reflectionless.

\section{Flow on a Torus}

The formula (3.9) recovers the potential $q(0)$ from spectral information of the operators $L(q)$ and $L_{D}(q)$. It suggests the following inverse spectral procedure; let $\left\{\mu_{j}(\xi)\right\}_{j=1}^{\infty}$ be the zeros of the Green's function $g(\xi, \xi ; \lambda)$ in the spectral gap $I_{j}$. These are eigenvalues of the half-line problems with Dirichlet conditions imposed at $x=\xi$. For reflectionless potentials the trace formula recovers $q(\xi)$ from $\left\{\mu_{j}\right\}$. In the next sections the motion of $\mu_{j}(\xi), \xi \in(-\infty, \infty)$ will be studied, and information about the class of reflectionless potentials will be deduced. We assume that $\sum_{j=1}^{\infty}\left|I_{j}\right|<\infty$ in the following; the conclusion will be that a reflectionless potential can be associated with a path on a torus. The torus is infinite dimensional if there are infinitely many spectral gaps; however, as $\left|I_{j}\right| \rightarrow 0$ as $j \rightarrow \infty$, the torus is compact.

The topology on the class of potentials $q \in C(\mathbb{R})$ is that of uniform convergence on compact sets. Define the hull of a potential $q(x)$ to be the closure with this topology of all translates of $q$ :

$$
\mathscr{H}(q)=\overline{\{q(x+\tau) ; \tau \in \mathbb{R}\}} .
$$

All $L(q(x+\tau))$ have the same spectrum, but limit points may not. The Weyl criterion allows us to draw some conclusions about the spectra of potentials in $\mathscr{H}(q)$.

Lemma 5.1. If $p \in \mathscr{H}(q)$ then either $\sigma(L(p))=\sigma(L(q))$ or $\sigma(L(p)) \subset \sigma_{\mathrm{ess}}(L(q))$. 
Proof. For $p \in \mathscr{H}(q)$ consider translates $\tau_{n}$ such that $q\left(x+\tau_{n}\right) \equiv q_{n}(x) \rightarrow p(x)$, uniformly on compact sets. If there is a subsequence $\tau_{n(m)} \rightarrow \tau \in \mathbb{R}$, then $p(x)=q(x+\tau)$ and $\sigma(L(p))=\sigma(L(q))$. If not, the sequence $\tau_{n}$ diverges, and we may assume it diverges monotonically to $\pm \infty$. For $\lambda \in \sigma(L(p))$ there exist approximate eigenfunctions $y_{m}(x) \in L^{2}(\mathbb{R})$ such that $\left\|y_{m}\right\|_{L^{2}}=1$ and $\left\|(L(p)-\lambda) y_{m}\right\|_{L^{2}}<2^{-m}$. Using a smooth cutoff function, we may even assume that $y_{m}(x)=0$ outside an interval $[-N(m), N(m)]$. Pick $n_{0}$ so large that on this interval $\left|q\left(x+\tau_{n}\right)-p(x)\right|<2^{-m}$ whenever $n \geqq n_{0}$. Then

$$
\begin{aligned}
\left\|\left(L\left(q_{n}\right)-\lambda\right) y_{m}\right\|_{L^{2}} & \leqq\left\|(L(p)-\lambda) y_{m}\right\|_{L^{2}}+\left\|\left(q_{n}-p\right) y_{m}\right\|_{L^{2}} \\
& \leqq 2^{1-m} .
\end{aligned}
$$

Certainly $n(m) \geqq n_{0}$ can be chosen sufficiently large that $\left|\tau_{n(m)}\right|>2 N(m)$. This constructs an orthonormal Weyl sequence $\left\{y_{m}\left(x-\tau_{n(m)}\right)\right\}_{m=1}^{\infty}$ for $\lambda$, for the potential $q(x)$, hence $\lambda \in \sigma_{\text {ess }}(L(q))$.

To obtain more information in the case of a reflectionless potential $q(x)$ and its hull, a technical lemma on the boundary behavior of Herglotz functions is used. This result has been used by other authors; for neatness it is included here.

Lemma 5.2. Consider a sequence of Herglotz functions $h_{n}(\lambda)$ which converges to some $h(\lambda)$ uniformly on compact sets of $\mathbb{C}^{+}$Let $A_{n}=\{\lambda \in \mathbb{R} ;$ the nontangential limit re $\left.h_{n}(\lambda+i 0)=0\right\}$. Suppose that there is a set in common $A=\bigcap_{n=1}^{\infty} A_{n}$. Then
re $h(\lambda)=0$ for almost every $\lambda \in A$.

Proof. There is a representation theorem for Herglotz functions in terms of the Cauchy integral. For $a \in \mathbb{R}, b \geqq 0$ and $e$ a nonnegative Borel measure on $\mathbb{R}$, such that $\int\left(1+\mu^{2}\right)^{-1} d e(\mu)<\infty$, the integral

$$
h(\lambda)=a+b \lambda+\int\left(\frac{1}{\mu-\lambda}-\frac{\mu}{1+\mu^{2}}\right) d e(\mu)
$$

defines a Herglotz function, and conversely each Herglotz function is represented by a unique $a, b$, and $e$. The measure $e$ is recovered from the nontangential limits of $\operatorname{im} h(\lambda)$, which are known to exist almost everywhere; if $I=\left(\lambda_{1}, \lambda_{2}\right)$, with $e\left(\left\{\lambda_{j}\right\}\right)=0$ then

$$
\begin{gathered}
e(I)=\lim _{\epsilon \rightarrow 0^{+}} \frac{1}{\pi} \int_{I} \operatorname{im} h(\mu+i \varepsilon) d \mu, \\
e_{\mathrm{ac}}(\mu)=\lim _{\epsilon \rightarrow 0^{+}} \frac{1}{\pi} \operatorname{im} h(\mu+i \varepsilon) .
\end{gathered}
$$

The uniqueness statement of this representation implies that if $h_{n}(\lambda) \rightarrow h(\lambda)$ uniformly on compact subsets of $\mathbb{C}^{+}$, then $a_{n} \rightarrow a, b_{n} \rightarrow b$ and $e_{n} \rightarrow e$ as Borel measures.

To prove Lemma 5.2 consider the representation of the Herglotz functions $\log h_{n}(\lambda) \rightarrow \log h(\lambda)$. The representation of $\log h(\lambda)$ is less singular than that for $h(\lambda)$ itself, since $0 \leqq$ im $\log h(\lambda) \leqq \pi$.

$$
\log h(\lambda)=a+b \lambda+\int\left(\frac{1}{\mu-\lambda}-\frac{\mu}{1+\mu^{2}}\right) d e(\mu),
$$


where $d e(\mu)=e_{\mathrm{ac}}(\mu) d \mu$ has no singular part. In fact, for test functions $f$ :

$$
\begin{aligned}
\int f(\mu) d e(\mu) & =\lim _{\epsilon \rightarrow 0} \frac{1}{\pi} \int f(\mu) \operatorname{im} \log h(\mu+i \varepsilon) d \mu \\
& =\frac{1}{\pi} \int f(\mu) \lim _{\epsilon \rightarrow 0} \operatorname{im} \log h(\mu+i \varepsilon) d \mu=\int f(\mu) e_{\mathrm{ac}}(\mu) d \mu,
\end{aligned}
$$

by the bounded convergence theorem. Thus, for any subset

$$
\begin{gathered}
B \subset A=\left\{\lambda ; \text { re } h_{n}(\lambda+i 0)=0\right\}, \\
\lim _{n \rightarrow \infty} \int_{B} d e_{n}(\mu)=\frac{1}{2} m(B)=\int_{B} d e(\mu),
\end{gathered}
$$

implying that $\arg h(\lambda+i 0)=\pi / 2$ for almost every $\lambda \in A$.

The Herglotz representation of $\log h(\lambda)$, when exponentiated, gives a product representation of $h(\lambda)$. The equality (5.2) shows that this product representation is less singular than the "partial fractions" representation, or usual Herglotz representation of (5.1).

This result is applied to the Green's function to obtain information about the hull of a reflectionless potential.

Lemma 5.3. Let $q(x)$ be reflectionless, and let $p(x) \in \mathscr{H}(q)$. Then $p(x)$ is also reflectionless, and $\sigma(L(p))=\sigma((L(q))-B$, where $m(B)=0$.

Proof. Let $q\left(x+\tau_{n}\right) \equiv q_{n}(x) \rightarrow p(x) \in \mathscr{H}(q)$. Consider the Herglotz functions $h_{n}(\lambda)=g_{n}(x, x ; \lambda)$ and $h(\lambda)=g(x, x ; \lambda)$, the Green's functions for $q_{n}, p$, respectively. If $A \subset \sigma(L(q))$, then $\operatorname{re} h_{n}(\lambda+i 0)=0$ for almost every $\lambda \in A$; hence, this property holds in the limit and $p(x)$ is reflectionless. Defining $B=\sigma(L(q))-\sigma(L(p))$, then (1) $g(x, x ; \lambda)$ is analytic at each $\lambda \in B$, (2) $\operatorname{im} g(x, x ; \lambda)=0$ for all $\lambda \in B$, and (3) by Lemma 5.2 , re $g(x, x ; \lambda)=0$ for almost every $\lambda \in B$. Thus, $m(B)=0$, for otherwise (1), (2), and (3) imply $g \equiv 0$.

It certainly can happen that the set $B$ is nonempty; however, if $\sigma(L(q))=\sigma_{\mathrm{ac}}(L(q))$ is entirely absolutely continuous, then for any set $A$ with $m(A)=0$,

$$
\overline{\sigma(L(q))-A}=\sigma(L(q)) .
$$

If in addition $q$ is reflectionless, Lemma 5.3 implies that the spectrum is constant for all $p \in \mathscr{H}(q)$. We have proved the following lemma.

Lemma 5.4. If $\sigma(L(q))=\sigma_{\mathrm{ac}}(L(q))$, then the spectrum is constant on $(q)$.

Lemmas 5.3 and 5.4 identify situations in which every point in the hull $\mathscr{H}(q)$ gives rise to the same torus. Lemma 5.4 appears in similar form in the work of Pastur [17] and Kotani [9]. If $P$ is an invariant ergodic probability measure on $\Omega$ giving rise to a class of ergodic potentials, then for all $q \in \operatorname{supp} P, \mathscr{H}(q) \subseteq \operatorname{supp} P$. Furthermore there are potentials $p \in \operatorname{supp} P$ such that $\mathscr{H}(p)=\operatorname{supp} P$.

A potential $q(x)$ which is reflectionless can be regarded as a function defined on a torus, evaluated along a path which is parameterized by $x$. This torus is most 
often infinite dimensional, and is associated with the gaps $\left\{I_{j}\right\}_{j=1}^{\infty}$ in the spectrum $\sigma(L(q))$. Define the torus in terms of coordinate charts; $T=\langle\boldsymbol{\mu}, \boldsymbol{\sigma}\rangle$ with

$$
\begin{array}{ll}
\boldsymbol{\mu}=\left\langle\mu_{1}, \mu_{2}, \ldots\right\rangle & \lambda_{2 j-1} \leqq \mu_{j} \leqq \lambda_{2 j} \\
\boldsymbol{\sigma}=\left\langle\sigma_{1}, \sigma_{2}, \ldots\right\rangle & \sigma_{j}= \pm 1 .
\end{array}
$$

One chart is given by specifying $\sigma$, and letting $\boldsymbol{\mu}$ range over the above values. Charts are connected by associating the sets $\left\{\mu_{j}=\lambda_{2 j}, \sigma_{j}=+1\right\}$ with $\left\{\mu_{j}=\lambda_{2 j}\right.$, $\left.\sigma_{j}=-1\right\}$, and similarly with $\lambda_{2 j-1}$. This still does not give a complete atlas for $T$; to give more global coordinates define constants

$$
\zeta_{j}=\left(\lambda_{2 j}-\lambda_{2 j-1}\right) / 2, \quad \xi_{j}=\left(\lambda_{2 j}+\lambda_{2 j-1}\right) / 2,
$$

and then globally define angles $\varphi$ by

$$
\begin{gathered}
\zeta_{j} \cos \varphi_{j}=\mu_{j}-\xi_{j}, \quad \lambda_{2 j-1} \leqq \mu_{j} \leqq \lambda_{2 j}, \\
0<\varphi_{j}<\pi \text { for } \sigma_{j}=+1, \quad \pi<\varphi_{j}<2 \pi \text { for } \sigma_{j}=-1 .
\end{gathered}
$$

There is no ambiguity in $\varphi_{j}$ when $\mu_{j}=\lambda_{2 j-1}$ or $\lambda_{2 j}$. The topology of $T$ is given by the distance functional

$$
d(\boldsymbol{\varphi}, \boldsymbol{\theta})=\sup _{j} d_{j}\left(\varphi_{j}, \theta_{j}\right), \quad d_{j}\left(\varphi_{j}, \theta_{j}\right)=\zeta_{j}^{1 / 2}\left|\varphi_{j}-\theta_{j}\right|_{\bmod 2 \pi}
$$

The assumption throughout this section is that $\Sigma \zeta_{j}<\infty$; this implies the compactness of $T$ with this topology.

We now define the map from a reflectionless potential to its torus, which is denoted by $\Phi ; q(x) \rightarrow \varphi$. Let $q(x) \rightarrow q(x+\xi)$ for $\xi \in \mathbb{R}$, and let $\mu_{j}(\xi) \in\left[\lambda_{2 j-1}, \lambda_{2 j}\right]$ be the zeros of $g(\xi, \xi ; \lambda)$ in the gap $I_{j}$ or an endpoint as above. As long as $\mu_{j}(\xi) \in\left(\lambda_{2 j-1}, \lambda_{2 j}\right)$, it is differentiable with respect to $\xi$, and satisfies a differential equation

$$
0=\frac{d}{d \xi} g\left(\xi, \xi ; \mu_{j}\right)=\partial_{\xi} g\left(\xi, \xi ; \mu_{j}\right)+\partial_{\lambda} g\left(\xi, \xi ; \mu_{j}\right) \frac{d \mu_{j}}{d \xi}
$$

The Green's function can be expressed in terms of the Weyl $m$-functions:

$$
\begin{gathered}
f_{ \pm}(x, \lambda)=\exp \left(\int_{0}^{x} m_{ \pm}(z, \lambda) d z\right), \\
g(x, y ; \lambda)=\frac{f_{+}(x, \lambda) f_{-}(y, \lambda)}{m_{-}-m_{+}}, \quad y \leqq x .
\end{gathered}
$$

Differentiating $g(x, x ; \lambda)$ in $x$, using $(5.7)$,

$$
\partial_{\xi} g(\xi, \xi ; \lambda)=\frac{m_{-}(\xi, \lambda)+m_{+}(\xi, \lambda)}{m_{-}(\xi, \lambda)-m_{+}(\xi, \lambda)} .
$$

Zeros $\mu \in I_{j}$ of the Green's function $g(\xi, \xi ; \lambda)$ correspond to poles of the Weyl functions $m_{ \pm}(\lambda)$. A pole of $m_{+}(\lambda)$ corresponds to an eigenvalue of $L(q)$ for the Dirichlet problem on $[\xi,+\infty)$, and correspondingly for $m_{-}(\lambda)$. Since $\lambda$ is not in the spectrum, they cannot both be singular. Evaluating (5.8) at the zero $\mu \in I_{j}$,

$$
\partial_{\xi} g(\xi, \xi ; \lambda)=\mp 1=-\sigma_{j}(\xi)
$$


where $\mp$ corresponds to the left or right half-line Dirichlet eigenvalue. This defines $(\boldsymbol{\mu}(\xi), \boldsymbol{\sigma}(\xi))$ as a point of $T$. The vector field on $T$ satisfied by $\boldsymbol{\mu}(\xi)$ is obtained from (5.6),

$$
\frac{d \mu_{j}}{d \xi}=\frac{\sigma_{j}(\xi)}{\partial_{\lambda} g\left(\xi, \xi ; \mu_{j}\right)} \equiv V_{j}(\mu, \sigma, \xi) .
$$

This so far is only defined in the individual charts (5.3). The next theorem discusses the behavior of $(\boldsymbol{\mu}(\xi), \boldsymbol{\sigma}(\xi))$ at gap endpoints as well.

Theorem 5.5. The translations $q(x) \rightarrow q(x+\xi)$ define a continuous path on the torus $T$. Specifically, $\mu_{j}(\xi) \in\left[\lambda_{2 j-1}, \lambda_{2 j}\right]$ is continuous in $\xi$, and $\sigma_{j}(\xi)$ is constant as long as $\mu_{j}(\xi) \in\left(\lambda_{2 j-1}, \lambda_{2 j}\right)$.

Proof. The pole $\mu_{j}(\xi) \in\left(\lambda_{2 j-1}, \lambda_{2 j}\right)$ is an isolated eigenvalue of a half-line Dirichlet problem, of multiplicity 1 . Under small perturbations $\xi \rightarrow \xi+\delta \xi$ this eigenvalue persists, and $\sigma_{j}$ remains constant. The difference quotient for $d \mu_{j} / d \xi$ converges, giving rise to the vector field (5.9). If $\mu_{j}(\xi)=\lambda_{2 j}$ (respectively $\lambda_{2 j-1}$ ), an endpoint, then $g(\xi, \xi ; \lambda)<0$ in $I_{j},(g(\xi, \xi ; \lambda)>0)$. For any $\lambda \in\left(\lambda_{2 j-1}+\varepsilon, \lambda_{2 j}-\varepsilon\right)$ express $g$ using Gauss' law of mean:

$$
\begin{aligned}
g(\xi, \xi ; \lambda) & =\frac{1}{\pi \varepsilon} \int_{S_{\varepsilon / 2} \lambda} \operatorname{reg} g(\xi, \xi ; \mu) d S_{\mu} \\
& =\frac{1}{\pi \varepsilon} \int_{S_{1}} \operatorname{re} g(\xi, \xi ; \mu) d S_{\mu}+\frac{1}{\pi \varepsilon} \int_{S_{2}} \operatorname{re} g(\xi, \xi ; \mu) d S_{\mu},
\end{aligned}
$$

with $S_{1}=S_{\varepsilon / 2}(\lambda) \cap\left\{\operatorname{im} \lambda>\varepsilon_{1}\right\}$ and $S_{2}=S_{\varepsilon / 2}(\lambda)-S_{1}$. The arcs of $S_{1}$ lie within $\mathbb{C}^{ \pm}$, where $g$ is continuous with respect to convergence of $q(x+\xi)$ uniformly on compact sets. On the arcs of $S_{2}$, there is an upper bound $|g(\xi, \xi ; \mu)|<C_{0} / \operatorname{dist}(\mu, \sigma(L(q)))$ which controls the contribution of the integral over $S_{2}$. Thus for small $\delta \xi, g(\xi+\delta \xi, \xi+\delta \xi ; \lambda)<0$ in $\left(\lambda_{2 j-1}, \lambda_{2 j}-\varepsilon\right)$, and $\mu_{j}(\xi)$ is continuous at the gap endpoints as well.

The topology of $T$ is such that the continuity of $(\mu(\xi), \sigma(\xi))$ follows from that of the individual $\mu_{j}(\xi)$, since for any $\varepsilon>0$ there are only finitely many $\zeta_{j}^{1 / 2} \geqq \varepsilon$, thus finitely many $\mu_{j}(\xi)$ to be considered.

We have obtained a result for reflectionless potentials that is familiar in the periodic case. The Dirichlet eigenvalues $\mu_{j}$ move in the gaps $I_{j}$ as the potential is translated. The motion is uniform in each gap, either increasing or decreasing monotonically, for $\partial_{\lambda} g(\xi, \xi ; \lambda)>0$ and $\sigma_{j}$ is constant as long as $\lambda_{2 j-1}<\mu_{j}<\lambda_{2 j}$. To continue thus analogy the Green's function, and the vector field (5.9) are considered in more detail.

At first glance the vector field (5.9) might depend explicitly on $\xi$, which would be bad for our purposes. The next lemma is thus useful.

Lemma 5.6. The vector field is autonomous;

$$
V_{j}(\mu, \sigma, \xi)=V_{j}(\mu, \sigma) .
$$


Proof. We again use the Herglotz representation of $\log g(\xi, \xi ; \lambda)$

$$
\begin{aligned}
\log g(\xi, \xi ; \lambda)-\log \sqrt{\lambda_{0}-\lambda}= & \frac{1}{\pi} \int_{-\infty}^{\infty} \frac{1}{v-\lambda} \\
& \times\left(\operatorname{im} \log g(\xi, \xi ; v+i 0)-\frac{\pi}{2} \chi_{\left[\lambda_{0},+\infty\right]}(v)\right) d v-\log 2 \\
= & \frac{1}{\pi} \int_{\lambda_{0}}^{\infty} \frac{1}{v-\lambda} \\
& \times\left(\operatorname{im} \log g(\xi, \xi ; v+i 0)-\frac{\pi}{2}\right) d v-\log 2 .
\end{aligned}
$$

We have used that the representing measure for $\log g$ is entirely absolutely continuous, Since $q(x)$ is reflectionless it is determined entirely by $\left\{\lambda_{j}\right\}_{j=0}^{\infty}$ and by the zeros $\left\{\mu_{j}\right\}_{j=1}^{\infty}$ of $g(\xi, \xi ; \lambda)$ in the spectral gaps,

$$
\begin{aligned}
\log g(\xi, \xi ; \lambda)-\log \sqrt{\lambda_{0}-\lambda} & =\frac{1}{\pi} \int_{\lambda_{0}}^{+\infty} \frac{1}{v-\lambda} \\
& \times \sum_{j=1}^{\infty}\left(\frac{\pi}{2} \chi_{\left(\lambda_{2 j}-1, \mu_{j}\right)}-\frac{\pi}{2} \chi_{\left(\mu_{j}, \lambda_{2 j}\right)}\right) d v-\log 2 \\
& =\sum^{\infty} \frac{1}{2}\left(\log \left(\frac{\mu_{j}-\lambda}{\lambda_{2 j-1}-\lambda}\right)+\log \left(\frac{\mu_{j}-\lambda}{\lambda_{2 j}-\lambda}\right)\right)-\log 2 .
\end{aligned}
$$

Finally, expression (5.10), whose dependence on $\boldsymbol{\mu}$ is explicit, is used to compute the vector field (5.9).

The Herglotz representation in (5.10) expresses a product representation of $g(\xi, \xi ; \lambda)$ for $\lambda \in \mathbb{C}^{+}$. An easy estimate shows that this representation can be continued into the spectral gaps. That is, for $\lambda \in I_{j}$,

$$
g(\xi, \xi ; \lambda)=\frac{1}{2} \frac{1}{\sqrt{\lambda_{0}-\lambda}} \prod_{j=1}^{\infty} \sqrt{\left(\frac{\lambda-\mu_{j}}{\lambda-\lambda_{2 j-1}}\right)\left(\frac{\lambda-\mu_{j}}{\lambda-\lambda_{2 j}}\right)},
$$

where the branch of the square root is taken which maps $\mathbb{C}-(-\infty, 0]$ into the right half-plane. The behavior of the representation at the endpoints $\lambda_{2 j-1}$ and $\lambda_{2 j}$ is more problematic, and will be taken up in several cases in the following section.

\section{A Compactness Theorem}

In the previous section a reflectionless potential is shown to be recovered by the evaluation of a function (the trace formula) along a continuous path on the torus $T$. In this section we derive some consequences of this inversion formula. In particular, we obtain information about $q(x)$ and $\mathscr{H}(q)$. We unfortunately cannot proceed for completely general closed sets $\sigma=\sigma(L(q))$; a geometrical condition will be imposed on the spectrum which will imply that (i) the spectrum is constant on $\mathscr{H}(q)$, and (ii) the vector field (5.9) is $C^{1}$ continuous on $T$. The conclusion is 
then that $\mathscr{H}(q)$ is compact. For simplicity we will exclude a discussion of isolated point spectrum, such as the eigenvalues of Bargmann potentials; these could be handled as well as long as accumulation points are excluded.

Formulae (5.8) and (5.11) describe the motion of $\mu_{j}(\xi) \in I_{j}$, which is interpreted as the motion of $(\boldsymbol{\mu}(\xi), \boldsymbol{\sigma}(\xi))$ on $T$ in coordinates,

$$
\begin{aligned}
\frac{d \mu_{j}}{d \xi}=V_{j}(\boldsymbol{\mu}, \boldsymbol{\sigma})= & 2 \sigma_{j} \sqrt{\left(\lambda_{0}-\mu_{j}\right)\left(\lambda_{2 j}-\mu_{j}\right)\left(\lambda_{2 j-1}-\mu_{j}\right)} \\
& \times \prod_{k \neq j} \sqrt{\left(\frac{\lambda_{2 k}-\mu_{j}}{\mu_{k}-\mu_{j}}\right)\left(\frac{\lambda_{2 k-1}-\mu_{j}}{\mu_{k}-\mu_{j}}\right) .}
\end{aligned}
$$

In the global angular coordinates $\varphi$ this equation is transformed into the equation

$$
\frac{d \varphi_{j}}{d \xi}=W_{j}(\varphi)
$$

Denote $\zeta_{j}=\left(\lambda_{2 j}-\lambda_{2 j-1}\right) / 2, \xi_{j}=\left(\lambda_{2 j}+\lambda_{2 j-1}\right) / 2$, and the distance between spectral gaps $\varrho_{j k}=\lambda_{2 j-1}-\lambda_{2 k}$ when $I_{k}<I_{j}$. From (5.11) the vector field has coefficients

$$
\begin{aligned}
W_{j}(\varphi)= & 2 \sqrt{\varrho_{j 0}+2 \zeta_{j} \cos ^{2}\left(\varphi_{j} / 2\right)} \\
& \times \prod_{I_{k}<I_{j}} \frac{\sqrt{\left(\varrho_{j k}+2 \zeta_{j} \cos ^{2}\left(\varphi_{j} / 2\right)\right)\left(\varrho_{j k}+\zeta_{k}+2 \zeta_{j} \cos ^{2}\left(\varphi_{j} / 2\right)\right)}}{\varrho_{j k}+2\left(\zeta_{j} \cos ^{2}\left(\varphi_{j} / 2\right)+\zeta_{k} \sin ^{2}\left(\varphi_{k} / 2\right)\right)} \\
& \times \prod_{I_{k}>I_{j}} \frac{\sqrt{\left(\varrho_{j k}+2 \zeta_{j} \sin ^{2}\left(\varphi_{j} / 2\right)\right)\left(\varrho_{j k}+\zeta_{k}+2 \zeta_{j} \cos ^{2}\left(\varphi_{j} / 2\right)\right)}}{\varrho_{j k}+2\left(\zeta_{j} \sin ^{2}\left(\varphi_{j} / 2\right)+\zeta_{k} \cos ^{2}\left(\varphi_{k} / 2\right)\right)}
\end{aligned}
$$

One simple advantage of these coordinates is that the bothersome choices of \pm 1 in different coordinate patches has been eliminated. We place geometrical conditions on the spectrum so that this vector field has a global $C^{1}$ bound on $T$; the condition takes into account both the sizes of gaps $I_{j}$, and their relative distances $\varrho_{j k}$. Define constants

$$
\left\|W_{j}(\varphi)\right\|_{L \infty(T)}=C_{j} .
$$

Lemma 6.1. The constants have an estimate

$$
C_{j} \leqq \sqrt{\varrho_{0 j}+\zeta_{j}} \exp \left(\frac{1}{2} \sum_{k \neq j} \zeta_{k} / \varrho_{j k}\right) .
$$

Proof. Setting $\mu_{j}=\zeta_{j} \cos \varphi_{j}+\xi_{j}$, one estimates

$$
\begin{aligned}
4\left|W_{j}(\varphi)\right|^{2} & =\left(\mu_{j}-\lambda_{0}\right) \prod_{k \neq j}\left(\frac{\lambda_{2 k}-\mu_{j}}{\mu_{k}-\mu_{j}}\right)\left(\frac{\lambda_{2 k-1}-\mu_{j}}{\mu_{k}-\mu_{j}}\right) \\
& \leqq\left|\mu_{j}-\lambda_{0}\right| \prod_{I_{k}<I_{j}}\left(1+\frac{\mu_{k}-\lambda_{2 k-1}}{\mu_{j}-\mu_{k}}\right) \prod_{I_{k}>I_{j}}\left(1+\frac{\lambda_{2 k}-\mu_{k}}{\mu_{k}-\mu_{j}}\right) \\
& \leqq\left(\varrho_{0 j}+\zeta_{j}\right) \exp \left(\sum_{k \neq j} \log \left(1+\zeta_{k} / \varrho_{j k}\right)\right) \\
& \leqq\left(\varrho_{0 j}+\zeta_{j}\right) \exp \left(\sum_{k \neq j} \zeta_{j} / \varrho_{j k}\right) .
\end{aligned}
$$


Theorem 6.2. If the following three geometrical conditions hold for the closed set $\sigma(L(q))$, then $\boldsymbol{W}(\boldsymbol{\varphi})$ is globally $C^{1}$ on $T$, and thus has global solutions $\varphi(\xi),-\infty<\xi<+\infty$.

(i) The measure of the gaps is finite;

$$
\sum_{j=1}^{\infty} \zeta_{j} \leqq C_{0}<+\infty
$$

(ii) The product is uniformly bounded in $j$;

$$
\zeta_{j} C_{j} \leqq C_{0}
$$

(iii) For convenience set $\zeta_{0}=1$. The sum is also uniformly bounded in $j$;

$$
\sum_{\substack{k=0 \\ k \neq j}}^{\infty} \frac{\zeta_{j}^{1 / 2} \zeta_{k}^{1 / 2}}{\varrho_{j k}} C_{j} \leqq C_{0}
$$

Proof. Condition (i) is already used in the trace formula, to recover limits as $t \rightarrow 0^{+}$of the Laplace transform of the trace of the difference of resolvants. It incidentally implies that $\zeta_{j} \rightarrow 0$ as $j \rightarrow \infty$. Condition (ii) implies firstly that each component of the vector field $\boldsymbol{W}$ is pointwise bounded. The vector field will be shown to be $C^{1}$ if the Frechet derivative $\partial_{\varphi} W$ exists as a bounded operator on the tangent space of $T$, with a uniform bound. The directional derivatives of the components of $\boldsymbol{W}$ are:

$$
\begin{aligned}
& \partial_{\varphi_{k}} W_{j}=\frac{1}{\mu_{k}-\mu_{j}} W_{j} \zeta_{k} \sin \varphi_{k}, \quad k \neq j \\
& \partial_{\varphi_{j}} W_{j}=\frac{1}{2}\left(\frac{1}{\mu_{j}-\lambda_{0}}+\sum_{k \neq j}\left(\frac{1}{\lambda_{2 k}-\mu_{j}}+\frac{1}{\lambda_{2 k-1}-\mu_{j}}-\frac{2}{\mu_{k}-\mu_{j}}\right)\right) W_{j} \zeta_{j} \sin \varphi_{j} .
\end{aligned}
$$

One estimates

$$
\begin{aligned}
\|W(\varphi)-W(\theta)\| & =\sup _{j} \zeta_{j}^{1 / 2}\left|W_{j}(\varphi)-W_{j}(\theta)\right| \\
& \leqq \sup _{j} \zeta_{j}^{1 / 2}\left(\sum_{k} \sup _{\psi \in T}\left|\partial_{\varphi_{k}} W_{j}(\psi)\right|\left|\varphi_{k}-\theta_{k}\right|\right) \\
& \leqq \sup _{j}\left(\sum_{k} \zeta_{j}^{1 / 2} / \zeta_{k}^{1 / 2}\left\|\partial_{\varphi_{k}} W_{j}\right\|_{L \infty(T)} \sup _{k} \zeta_{k}^{1 / 2}\left|\varphi_{k}-\theta_{k}\right|\right) \\
& =\sup _{j}\left(\sum_{k} \frac{\zeta_{j}^{1 / 2}}{\zeta_{k}^{1 / 2}}\left\|\partial_{\varphi_{k}} W_{j}\right\|_{L \infty(T)}\right)\|\varphi-\theta\| .
\end{aligned}
$$

Split the remaining sum in two; the first term can be estimated

$$
\sup _{j}\left(\sum_{k \neq j} \frac{\zeta_{j}^{1 / 2}}{\zeta_{k}^{1 / 2}}\left\|\partial_{\varphi_{k}} W_{j}\right\|_{L \infty(T)}\right) \leqq \sup _{j}\left(\sum_{k} \frac{\zeta_{j}^{1 / 2} \zeta_{k}^{1 / 2}}{\varrho_{j k}}\left\|W_{j}\right\|_{L \infty(T)}\right) \leqq C_{0},
$$


if the geometrical condition (iii) holds for the set $\sigma(L(q))$. The remaining term in the sum has a similar estimate. Rewrite

$\frac{1}{\lambda_{2 k}-\mu_{j}}+\frac{1}{\lambda_{2 k-1}-\mu_{j}}-\frac{2}{\mu_{k}-\mu_{j}}=\frac{\mu_{k}-\lambda_{2 k}}{\left(\lambda_{2 k}-\mu_{j}\right)\left(\mu_{k}-\mu_{j}\right)}+\frac{\mu_{k}-\lambda_{2 k-1}}{\left(\lambda_{2 k-1}-\mu_{j}\right)\left(\mu_{k}-\mu_{j}\right)}$.

Then from (6.5),

$$
\sup _{j}\left\|\partial_{\varphi_{j}} W_{j}\right\|_{L \infty(T)} \leqq \sup _{j} \frac{1}{2}\left(\frac{\zeta_{j}}{\varrho_{0 j}}+\sum_{k \neq j} 2 \frac{\zeta_{j} \zeta_{k}}{\varrho_{j k}^{2}}\right)\left\|W_{j}\right\|_{L \infty(T)} .
$$

Estimating $\sum_{k \neq j} \frac{\zeta_{j} \zeta_{k}}{\varrho_{j k}^{2}} \leqq\left(\sum_{k \neq j} \zeta_{j}^{1 / 2} \zeta_{k}^{1 / 2} / \varrho_{j k}\right)^{2}$, the geometrical condition (iii) implies that

$$
\sup _{j}\left\|\partial_{\varphi_{j}} W_{j}\right\|_{L \infty(T)} \leqq 3 C_{0}^{2} .
$$

This finishes the $C^{1}$ estimate. Condition (i) is in fact implied by condition (iii); it is retained for its intuitive content.

Denote by $\mathscr{C}$ the class of closed set for which (6.4) (i) (ii) (iii) hold. These conditions do not exclude Cantor sets, as we shall see below. The conditions can be violated, however, by very tight accumulation of large gaps, with asymptotically very small distance between the gaps. Some examples of sets $\sigma$ satisfying (i) (ii) (iii) are given here.

1. If $\Sigma \zeta_{j}<\infty$, and the gap endpoints $\lambda_{j}$ are distinct and accumulate at most only at $+\infty$, then (6.4) holds. This includes spectra of all periodic potentials $q(x)$, as well as the Dubrovin, Mateev, and Novikov examples [6] of finite gap spectra.

2. Limit periodic potentials of the type constructed by Moser [16], Avron and Simon [1] and others. These possess Cantor spectra which are nowhere dense and of large Lebesgue measure, and the spectral gaps accumulate in such a way that conditions (6.4) are satisfied.

3. The reflectionless potentials of Bargmann do not have a global $C^{1}$ bound, but this is due only to a singular set of $\boldsymbol{W}$ on $T$ of codimension 2 . This arises from the fact that they are limiting cases of finite dimensional tori, where the dimensionality is dropping by 1 .

4. In two papers [11,12], Levitan constructed almost periodic potentials given through orbits on infinite dimensional tori. The trace formula is also recovered. In [10] Kotani and Krishna improved upon this work, allowing more complicated spectra. Under their hypotheses, it can be shown that $\boldsymbol{W}(\varphi)$ is $C^{1}$ in the $l^{2}$ topology on $T$;

$$
\|\varphi-\theta\|_{l^{2}}^{2}=\sum_{j=1}^{\infty} \zeta_{j}\left|\varphi_{j}-\theta_{j}\right|_{\bmod 2 \pi}^{2}
$$

Conditions on the set $\sigma(L(q))$, however, exclude nowhere dense or Cantor-like spectra.

5. A standard Cantor set of zero measure will violate condition (6.4) (iii). 
Proposition 6.3. If $\sigma \in \mathscr{C}$, then for any $B$ with $m(B)=0, \overline{\sigma-B}=\sigma$. Thus by Lemma 5.3 if $\sigma(L(q)) \in \mathscr{C}$, then $\sigma(L(p))=\sigma(L(q))$ for all $p \in \mathscr{H}(q)$.

Proof. It sufficies to show that if there is an interval $I$ such that $A \equiv \sigma \cap I \neq \phi$, and $m(A)=0$, then (6.4) (iii) is violated. First, we need all $\varrho_{j k}>0$ or else there is no hope of a uniform bound $C_{0}$. Label all intervals $I_{j} \leqq A^{\mathrm{C}} \cap I_{\text {; }}$ there must be infinitely many of them. Since $m(A)=0, \varrho_{j k}=\sum_{I_{j}<I_{l}<I_{k}} \zeta_{l}$. Consider $I_{j}$ fixed and
estimate

$$
\zeta_{j} \sum \frac{\zeta_{k}}{\varrho_{j k}^{2}} \geqq \zeta_{j} \sum_{\substack{j \neq k \\ I_{k} \cup A^{c} \cap I}} \frac{\zeta_{k}}{\left(\sum_{I_{k}<I_{l}<I_{j}} \zeta_{l}\right)^{2}} \geqq \zeta_{j} \sum_{I_{k}<I_{l}<I_{j}}\left(\frac{\zeta_{l}}{\left(\sum_{I_{k}<I_{l}<I_{j}} \zeta_{l}\right)^{2}}\right)=\zeta_{j} / \varrho_{j k}
$$

Taking $I_{k} \rightarrow I_{j}$ results in $\varrho_{j k} \rightarrow 0$, violating any bound $C_{0}$ attempted for (6.4) (iii).

6. A Cantor set of sufficiently positive measure will satisfy (6.4). Specifically, the set $\bigcup_{l=1}^{\infty} B_{l} \in \mathscr{C}$, where $B_{l}=$ a Cantor set of measure $m_{l}$ in the interval $[l-1, l], l \in \mathbb{Z}^{+}$, which is constructed in the usual way by excising evenly spaced intervals. If $\sum_{l=1}^{\infty} l\left(1-m_{l}\right)=C_{1}<\infty$ and each $m_{l}>6 / 7$, then the resulting torus will have a global $C^{1}$ bound for the vector field $W(\varphi)$.

To prove that the Cantor set described above gives rise to a $C^{1}$ vector field (6.2), we show that (6.4) (i) (ii) (iii) are satisfied. Certainly $\sum_{j=1}^{\infty} \zeta_{j} \leqq \sum_{l=1}^{\infty}\left(1-m_{l}\right) \leqq C_{1}$, so that (i) holds. Conditions (ii) and (iii) both depend upon control of the growth of $C_{j}$ as $j \rightarrow \infty$. The problem is basically for intervals $I_{k}$ with $\varrho_{j k}<1$, for otherwise the estimate reduces to that of (i). This Cantor set is constructed by recursively excising evenly spaced intervals of length $e^{-b k}, k=1,2, \ldots$. At step $k$ there are $2^{k}$ intervals excised of this length. Fix a gap $I$ from step $j$. For $k \geqq j$ the distance between any gap from step $k$ and $I$ is very close to $\varrho_{j k}=2^{-k}$, as long as at each step the excisions are spaced evenly within the remaining closed sets. For $k<j$ there are at most two worse cases: the nearest gap on the left and right of $I$; these can have $\varrho_{j k}=2^{-j}$ while have relatively large length $e^{-b k}$. All others at step $k<j$ have better distances $\varrho_{j k} \geqq 2^{-k}$. To estimate $C_{j}$

$$
\begin{aligned}
C_{j}^{2} & \leqq\left(\varphi_{0 j}+\zeta_{j}\right) \prod_{\substack{\text { two worse } \\
\text { cases }}}\left(1+\frac{\zeta_{k}}{\varphi_{j k}}\right) \exp \left(\sum_{\substack{\text { all other } \\
\text { cases }}} \frac{\zeta_{k}}{\varphi_{j k}}\right) \\
& \leqq\left(\varphi_{0 j}+1\right) 4 \exp (-b+j \log 2) \exp \left(\sum_{k=1}^{\infty} e^{-b k} e^{2 \log 2 k}\right) \\
& \leqq 4(l+1) \exp (j \log 2) \exp \left(\frac{1}{b-2 \log 2}\right)
\end{aligned}
$$

This grows at most exponentially in $j$. The factor $l$ is here because we are inspecting Cantor sets near $[l-1, l]$. Since $\zeta_{j}=|I|=\exp \left(-b_{j}\right), b>2 \log 2$, the bound on $C_{j} \zeta_{j}$ depends only upon $l$. Computing the measure of the Cantor set in $[l-1, l]$, 
$m_{l}=1-(\exp (b-\log 2)-1)^{-1}$, so that $b=b_{l} \sim-\log \left(1-m_{l}\right)$. If $l\left(1-m_{l}\right) \leqq C_{1}$, there is a global bound for $C_{j} \zeta_{j}$, and estimate (ii) is satisfied. Similar considerations give estimate (iii), where it is required that $b>4 \log 2$. The measure of these permitted sets is computed from this requirement of $b$, finding that $m_{l}>6 / 7$.

To have a selection criterion among closed sets $\sigma \subset \mathbb{R}$, in order to distinguish better the class $\mathscr{C}$, there is a comparison theorem. Define a partial order among closed sets of $\mathbb{R} ; \sigma_{1} \prec \sigma_{2}$ if every component $I^{1} \subseteq \mathbb{R}-\sigma_{1}$ intersects at most one component $I^{2} \subseteq \mathbb{R}-\sigma_{2}$, which it contains; $I^{2} \subseteq I^{1}$.

Theorem 6.4. If $\sigma_{1} \in \mathscr{C}$ and $\sigma_{1} \prec \sigma_{2}{ }^{\prime}$ then $\sigma_{2} \in \mathscr{C}$.

Proof. Estimates (6.4) (i) (ii) (iii) are all clearly monotone in $\sigma$, with respect to the partial order.

A global $C^{1}$ bound for the vector field $\boldsymbol{W}(\varphi)$ on $T$ implies the existence of global, unique integral curves, which for finite $\xi \in \mathbb{R}$ depend continuously on the initial data. When the spectrum $\sigma(L(q))$ of a reflectionless potential $q(x)$ is in class $\mathscr{C}$, more global information is obtained about $\mathscr{H}(q)$. The angles $\varphi_{j}(\xi)$ derived from translating the potential satisfy Eq. (6.2) as long as $\varphi_{j} \neq 0, \pi$; that is, as long as $\mu_{j}$ is not a gap endpoint. However, continuous $\varphi_{j}(\xi)$ which are solutions for almost every $\xi$ are a priori solutions for all $\xi$; thus the angles and the integral curves coincide. This is as in the periodic case, the auxiliary spectrum $\mu_{j}(\xi)$ touches a gap endpoint, and then reverses its direction without stopping.

Lemma 6.5. For $q(x)$ reflectionless, such that $\sigma(L(q)) \in \mathscr{C}$, the mapping $\Phi$ extends continuously to the hull $\mathscr{H}(q)$. That is, whenever $q^{n}(x)=q\left(x+\tau_{n}\right) \rightarrow p(x) \in \mathscr{H}(q)$, then

$$
\Phi(p(x))=\lim _{n \rightarrow \infty} \Phi\left(q^{n}(x)\right) \in T .
$$

Thus $\Phi$; $\mathscr{H}(q) \rightarrow T$ gives a continuous mapping of $\mathscr{H}(q)$ into a subset of $T$.

Proof. Since $\sigma(L(q)) \in \mathscr{C}$, Lemma 5.3 and Proposition 6.3 imply that the torus is constant over $\mathscr{H}(q)$. Techniques similar to those of the proof of Theorem 5.5 show that the points $\mu_{j}\left(q^{n}\right) \rightarrow \mu_{j}(p)$ as $q^{n}(x) \rightarrow p(x)$ in the topology of uniform convergence on compact sets.

We wish additionally to show that the image $\Phi(\mathscr{H}(q)) \subseteq T$ is a closed set; this will imply the compactness of $\Phi(\mathscr{H}(q))$.

Lemma 6.6. Let $\sigma(L(q)) \in \mathscr{C}$. If $\boldsymbol{\varphi}_{n}=\boldsymbol{\varphi}\left(\tau_{n}\right) \rightarrow \boldsymbol{\varphi}$ in $\Phi(\mathscr{H}(q)) \subseteq T$, then the orbit $\boldsymbol{\varphi}_{n}(\xi)=\boldsymbol{\varphi}\left(\xi+\tau_{n}\right)$ of (6.2) through $\varphi_{n}$ converges uniformly to $\varphi(\xi)$ for $\xi$ within compact subsets of $\mathbb{R}$.

Proof. This is just the results of continuous dependence of solutions of (6.2) on initial data.

Consider angles $\varphi_{n}=\Phi\left(q^{n}(x)\right)$ converging to a limit in $T$. For each $n$ we recover the potential via the trace formula

$$
q^{n}(\xi)=q\left(\xi+\tau_{n}\right)=\lambda_{0}+\sum_{j=1}^{\infty} \lambda_{2 j}+\lambda_{2 j-1}-2 \mu_{j}^{n}(\xi),
$$


with $\mu_{j}^{n}(\xi)=\zeta_{j} \cos \varphi_{j}^{n}(\xi)+\xi_{j}$. This expression is continuous with respect to the topology on $T$. Lemma 6.6 implies that $q^{n}(\xi)$ converges uniformly on compact sets to the function

$$
p(\xi)=\lambda_{0}+\sum_{j=1}^{\infty} \lambda_{2 j}+\lambda_{2 j-1}-2 \mu_{j}(\xi), \quad \mu_{j}(\xi)=\zeta_{j} \cos \varphi_{j}(\xi)+\xi_{j} ;
$$

thus $p \in \mathscr{H}(q)$. We have recovered the potential $p$ from spectral information $\left\{\varphi_{j}(\xi)\right\}_{j=1}^{\infty}$, including that $\sigma(L(q))=\sigma(L(p))$, and that the Green's function for $p(x)$ is given by

$$
g(x, x ; \lambda)=g(\boldsymbol{\mu}(x), \lambda),
$$

with the full Green's function obtainable from the diagonal by quadrature. This defines the inverse of the map $\Phi ; \mathscr{H}(q) \rightarrow T$ on the image $\Phi(\mathscr{H}(q)) \subseteq T$, as being continuous one-one, and onto $\mathscr{H}(q)$. That is, for $\varphi \in \Phi(\mathscr{H}(q))$ define (1) $\varphi(\xi)$ the integral curve of (6.2), (2) $\boldsymbol{\mu}(\xi)=\left\langle\zeta_{j} \cos \varphi_{j}+\xi_{j}\right\rangle_{j=1}^{\infty}$, (3) via the trace formula $p(\xi)=\lambda_{0}+\sum_{j=1}^{\infty} \lambda_{2 j}+\lambda_{2 j-1}-2 \mu_{j}(\xi)$; this inverts the map $\Phi$ on the image in $T$ of $\mathscr{H}(q)$. We have shown the result:

Theorem 6.7. For $q(x)$ reflectionless, with $\sigma(L(q)) \in \mathscr{C}$ then $\mathscr{H}(q)$ is compact, the continuous image under the trace formula of $\Phi(\mathscr{H}(q))$ a compact subset of $T$.

We expect that a reflectionless potential $q(x)$ with the assumption that $\sigma(L(q)) \in \mathscr{C}$, will be in fact an almost periodic function. The result of Theorem (6.7) is that $\mathscr{H}(q)$ is compact in the topology of uniform convergence on compact sets. If we knew the stronger result of compactness in the uniform topology this would already imply almost periodicity. This is a more delicate question, and requires detailed knowledge of the orbits of the vector field (6.2) on $T$.

We also expect that if $q(x)$ is an ergodic potential, then $\sigma(L(q))=\sigma_{\mathrm{ac}}(L(q))$ implies that in fact $q(x)$ is almost periodic. Theorem (6.7) gives the weaker result that if $q(x)$ is ergodic, then either (1) there is a subset $B$ of the spectrum of positive measure such that $\gamma(\lambda)>0$ on $B$, or (2) supp $P$ is compact, homeomorphic to a closed subset of a compact torus, or (3) the spectrum is an irregular set; that is $\sigma(L(q)) \notin \mathscr{C}$.

\section{Elliptic Spherical Coordinates}

This last section is to point out formal analogies between the orbits the orbits $\varphi(\xi) \in T$, and a mechanical problem of constrained harmonic oscillators first studied by C. Neumann. The connection between this problem and the Schrödinger operator was discovered by J. Moser and E. Trubowitz [15], in the setting of periodic potentials or finite gap potentials. We phrase their results here in terms of the Green's function, and we consider the general reflectionless case. In the ergodic setting, Kotani and Krishna [10] have discussed a similar analogy.

From Eq. (5.8), sometimes known as the Dubrovin equation, the motion of $\mu_{j}$ in a spectral gap is described,

$$
\frac{d \mu_{j}}{d \xi}=\frac{\sigma_{j}}{\partial_{\lambda} g\left(\boldsymbol{\mu}, \mu_{j}\right)} .
$$


The notation for the Green's function is, following Lemma 5.6, that $g(\xi, \xi ; \lambda) \equiv g(\boldsymbol{\mu}(\xi) \lambda)$, while $\sigma_{j}= \pm 1$ as before. We define a normalizing factor $\Gamma(\lambda)$ for what follows; this depends only upon the torus $T$, and as well $-\Gamma(\lambda)$ is a Herglotz function. For $\operatorname{im} \lambda>0$,

$$
\begin{aligned}
\Gamma(\lambda) & =\frac{1}{\sqrt{\lambda_{0}-\lambda}} \exp \left(-\frac{1}{2} \int_{-\infty}^{\infty} \frac{1}{v-\lambda}\left(\sum_{j=1}^{\infty} \chi_{\left(\lambda_{2 k-1}, \lambda_{2 k}\right)}\right) d v\right) \\
& =\frac{1}{\sqrt{\lambda_{0}-\lambda}} \prod_{j=1}^{\infty} \sqrt{\left(\frac{\lambda-\lambda_{2 k-1}}{\lambda-\lambda_{2 k}}\right)} .
\end{aligned}
$$

When the total gap length is finite, $\sum_{j=1}^{\infty} \zeta_{j}<\infty$, this representation can be continued into the open gaps. Following Moser [15], define a metric by giving diagonal entries

$$
g_{j}(\boldsymbol{\mu})=\frac{1}{4} \Gamma\left(\mu_{j}\right) \partial_{\lambda} g\left(\boldsymbol{\mu}, \mu_{j}\right)
$$

for $\mu_{j} \in I_{j}$, setting off diagonal entries to zero. This has a formal interpretation as the Euclidian metric restricted to the unit sphere, given in elliptic coordinates. The kinetic energy of (7.1) is given by

$$
2 T=\sum_{j=1}^{\infty} g_{j}(\boldsymbol{\mu})\left(\frac{d \mu_{j}}{d \xi}\right)^{2}
$$

although the expression does not necessarily converge. However, from (7.1) the law of conservation of energy is satisfied

$$
\frac{1}{2} \sum_{j=1}^{\infty} g_{j}(\boldsymbol{\mu})\left(\frac{d \mu_{j}}{d \xi}\right)^{2}-g_{j}(\boldsymbol{\mu})\left(\frac{1}{\partial_{\lambda} g\left(\boldsymbol{\mu}, \mu_{j}\right)}\right)^{2}=0,
$$

with potential energy

$$
U(\boldsymbol{\mu})=-\frac{1}{2} \sum_{j=1}^{\infty} \frac{1}{2} \frac{\Gamma\left(\mu_{j}\right)}{\partial_{\lambda} g\left(\boldsymbol{\mu}, \mu_{j}\right)} .
$$

From (7.5), the system can be guaranteed to have finite energy if the sum converges;

$$
8 U\left(\mu_{j}\right)=\sum_{j=1}^{\infty} \frac{\Gamma\left(\mu_{j}\right)}{\partial_{\lambda} g\left(\boldsymbol{\mu}, \mu_{j}\right)} .
$$

For certain spectral geometries this may be infinite; however, in the notation of Sect. 6 , if $\sum_{j=1}^{\infty} \zeta_{j}^{1 / 2} C_{j}<+\infty$, the potential energy, and hence also the kinetic energy, are finite on the torus. For spectra of class $\mathscr{C}$ this condition is satisfied.

The variables $\mu_{j}, d \mu_{j} / d \xi$ are Lagrangian variables. To obtain a Hamiltonian system, one defines canonical conjugate variables

$$
v_{j}=\partial_{\mu_{j}} T=g_{j}(\boldsymbol{\mu}) \frac{d \mu_{j}}{d \xi},
$$


which gives

$$
2 T=\sum_{j=1}^{\infty} \frac{1}{g_{j}} v_{j}^{2}=\sum_{j=1}^{\infty} \frac{4}{\Gamma\left(\mu_{j}\right) \partial_{\lambda} g\left(\boldsymbol{\mu}, \mu_{j}\right)} v_{j}^{2} .
$$

The Hamiltonian $H(\boldsymbol{\mu}, \boldsymbol{v})=T+U$ has formally the structure of a completely integrable system in infinitely many degrees of freedom. To prove complete integrability analytically, it would suffice to construct a smooth canonical transformation of the system to action-angle variables. From another point of view, this was carries out by Levitan $[11,12]$, for a class of spectra somewhat weaker than $\mathscr{C}$. The complete class of spectra for which this can be carried out, and for which orbits are shown to be almost periodic, is of great interest, and is yet to be fully explored.

Acknowledgements. I would like to thank many people for their interest in this work, in particular M. Krishna, V. Papanicolaou, and S. Venakides for useful discussions on the subject matter, and especially S. Kotani and J. Ralston for comments and corrections to a preprint of this paper.

\section{References}

1. Avron, J., Simon, B.: Almost periodic Schrödinger operators. I. Limit periodic potentials. Commun. Math. Phys. 82, 101-120 (1981)

2. Bargmann, V.: Remarks on the determination of the central field of force from the elastic scattering phase shift. Phys. Rev. 75, 301-303 (1949)

3. Craig, W.: Floquet exponents for Jacobi fields (preprint)

4. Deift, P., Trubowitz, E.: Inverse scattering on the line. Commun. Pure Appl. Math. 32, 121-251 (1979)

5. Douady, A., Hubbard, J.H., Verdier, J.L.: Equation de Hill Periodique. Seminaire de Geometrie Analytique de l'Ecole Normale Superiure 1976-77, seminar of A. Douady. Lecture notes

6. Dubrovin, A.A., Matveev, V.B., Novikov, S.P.: Nonlinear equations of Kortweg deVries type, finite zone linear operators and Abelian varieties. Russ. Math. Surv. 31, 59-146

7. Goldsheid, I., Molchanov, S., Pastur, L.: A pure point spectrum of the stochastic and onedimensional Schrödinger equation. Funct. Anal. Appl. 11, 1-10 (1977)

8. Johnson, R., Moser, J.: The rotation number for almost periodic potentials. Commun. Math. Phys. 84, 403-438 (1982) erratum: Commun. Math. Phys. 90, 317-318 (1983)

9. Kotani, S.: One-dimensional random Schrödinger operators and Herglotz functions. Proc. Taniguchi Symp. on Prob. Methods in Math Physics, Katata (to appear)

10. Kotani, S., Krishna, M.: Almost periodicity of some random potentials. J. Funct. Anal. 78 (1988)

11. Levitan, B. M.: Almost periodicity of infinite zone potentials. Math. USSR Isv. 18, 249-273 (1982)

12. Levitan, B.M.: Approximation of infinite zone potentials by finite zone potentials. Math. USSR Isv. 20, 55-87 (1983)

13. McKean, H., van Moerbeke, P.: The spectrum of Hill's equation. Invent. Math. 30, 217-274 (1975) 
14. McKean, H., Trubowitz, E.: Hill's operator and hyperelliptic function theory in the presence of infinitely many branch points. Commun. Pure Appl. Math. 29, 143-226 (1976)

15. Moser, J.: Integrable Hamiltonian systems and spectral theory. Lezioni Fermiane. Academia Nazionale dei Lincei - Scuola Normale Superiore - Pisa 1981

16. Moser, J.: An example of a Schrödinger operator with almost periodic potential and nowhere dense spectrum. Comment. Math. Helv. 56, 198-224 (1981)

17. Pastur, L.: Spectral properties of disordered systems in one-body approximation. Commun. Math. Phys. 75, 167-196 (1980)

18. Trubowitz, E.: The inverse problem for periodic potentials. Commun. Pure Appl. Math. 30, 321-337 (1977)

Communicated by T. Spencer

Received February 23, 1989; in revised from May 4, 1989 
